\title{
Kinds Are Calling Conventions
}

PAUL DOWNEN and ZENA M. ARIOLA, University of Oregon, USA

SIMON PEYTON JONES, Microsoft Research, UK

RICHARD A. EISENBERG, Bryn Mawr College, USA and Tweag I/O, UK

A language supporting polymorphism is a boon to programmers: they can express complex ideas once and reuse functions in a variety of situations. However, polymorphism is pain for compilers tasked with producing efficient code that manipulates concrete values.

This paper presents a new intermediate language that allows for efficient static compilation, while still supporting flexible polymorphism. Specifically, it permits polymorphism over not only the types of values, but also the representation of values, the arity of primitive machine functions, and the evaluation order of arguments-all three of which are useful in practice. The key insight is to encode information about a value's calling convention in the kind of its type, rather than in the type itself.

CCS Concepts: • Software and its engineering $\rightarrow$ Semantics; Compilers.

Additional Key Words and Phrases: arity, levity, representation, polymorphism, type systems

\section{ACM Reference Format:}

Paul Downen, Zena M. Ariola, Simon Peyton Jones, and Richard A. Eisenberg. 2020. Kinds Are Calling Conventions. Proc. ACM Program. Lang. 4, ICFP, Article 104 (August 2020), 29 pages. https://doi.org/10.1145/ 3408986

\section{INTRODUCTION}

Polymorphism supports reuse by allowing one piece of code to work with values of many different types. But ubiquitous polymorphism usually comes with a runtime cost: all values must share a common representation, usually a pointer to a "boxed" (heap-allocated) object. This is sometimes much less efficient than a monomorphic version of the same code, specialized to a particular representation (such as an unboxed 64-bit word).

One approach is to specialize code to a single type. But we would get more reuse if we could specialize to, say, "any type represented by an unboxed 64-bit word." Since kinds classify types, perhaps we can write code that is monomorphic in the kind, but polymorphic in the type. Hence our slogan: kinds are calling conventions. For example, consider the function twice:

$$
\text { twice } f x=f(f x)
$$

To control performance, we would like to have a say in matters like: Can $f$ be a thunk? How many arguments does $f$ expect (its arity)? Can $x$ be a thunk? How is $x$ represented? Moreover, we want to express the answers to these questions in a type system with strong static guarantees.

A major insight of this paper is the discovery that we can refine the vague notion of "ways in which we want to classify types" along three different axes:

Authors' addresses: Paul Downen; Zena M. Ariola, University of Oregon, Eugene, Oregon, USA, pdownen@cs.uoregon. edu, ariola@cs.uoregon.edu; Simon Peyton Jones, Microsoft Research, Cambridge, UK, simonpj@microsoft.com; Richard A. Eisenberg, Bryn Mawr College, Bryn Mawr, PA, USA, Tweag I/O, Cambridge, UK, rae@richarde.dev.

This work is licensed under a Creative Commons Attribution 4.0 International License.

(C) 2020 Copyright held by the owner/author(s).

2475-1421/2020/8-ART104

https://doi.org/10.1145/3408986 
- Representation. How is this argument represented at runtime?

- Levity. What is the evaluation strategy of this argument (e.g., call-by-value or call-by-need)?

- Arity. For functions, how many arguments are needed before its code can be executed?

Many functions can be polymorphic in some of these axes, but not in others.

Our focus is on an intermediate language. The programmer may write in a uniform language, but the compiler needs an intermediate language that can express low-level representation choices, and expose those choices to the optimizer. For example, the programmer might work exclusively with boxed integer values, say of type Int, but the intermediate language can have an unboxed type Int\#, together with explicit operations to box and unbox integers. This allows the optimizer to eliminate many box-followed-by-unbox chains [Peyton Jones and Launchbury 1991].

This paper builds directly on several earlier works that statically keep track of different representations [Eisenberg and Peyton Jones 2017] and function arities [Downen et al. 2019] within a type and kind system. Our new contribution is to bring them together into a single framework, more powerful and more precise than any of its predecessors. Specifically:

- We introduce a polymorphic intermediate language that statically captures calling conventions in kinds, and has polymorphism over the representation, levity, and arity of types (Section 4).

- Our intermediate language is equally well-suited for both eager and lazy functional languages. Concretely we show how to compile two higher-level, polymorphic source languages-callby-name and call-by-value System F-to our intermediate language (Section 5).

- We show how to compile our polymorphic intermediate language to a lower-level language with multiple representations (e.g., pointers versus integers) and multi-arity functions, but not polymorphism (Section 6). Compilation is driven by kinds and keeps type erasure and code reuse; typing restrictions ensures polymorphic code is compiled to monomorphic code.

- We provide evidence of correctness for the full compilation process (Theorems 1 to 4 ) from both call-by-name and call-by-value source languages to machine code.

- We describe a small extension to our intermediate language to allow for dynamic checks on the arities of closures, so that we can use the best arity available at runtime (Section 7).

\section{FUNCTION ARITY}

We identify three axes of classification, above. Of these three, we peel off arity to explain it first; mixed representations and evaluation strategies have existed for longer and may be more familiar. This section gives a high-level overview of our approach; the details will be nailed down in Section 4 .

\subsection{What Is Arity?}

What is a function's "arity?" In practical terms, the arity of a function determines what code a compiler will generate to call that function. All modern architectures support calling conventions that allow for efficiently calling subroutines by passing multiple arguments at once. If a function has arity $n$, then a call to that function will pass $n$ arguments simultaneously.

In a naively compiled curried language, every function has arity 1 . This implementation of functional languages is unacceptably inefficient. Instead, compilers must somehow map curried surface-language functions onto multi-argument machine-language functions.

To do this, we need two things. First, an arity analysis; and second, an intermediate language in which arity is explicit, so that we can express and memorialise the results of the analysis.

Arity analysis is not always straightforward. For example:

$$
\begin{array}{ll}
f_{1}, f_{2}, f_{3}: \text { Int } \rightarrow \text { Int } \rightarrow \text { Int } & \\
f_{1}=\lambda x . \lambda y . \text { let } z=\text { expensive } x \text { in } y+z & f_{2}=\lambda x \cdot f_{1} x \\
f_{3}=\lambda x . \text { let } z=\text { expensive } x \text { in } \lambda y \cdot y+z & f_{4}=\lambda x \cdot f_{3} x
\end{array}
$$


Here, $f_{1}$ clearly has arity 2 because it starts with two $\lambda$ s: it takes two arguments before computing a result. Function $f_{2}$ has only one $\lambda$, but still has arity 2 , because we could safely $\eta$-expand it to have two $\lambda$ s. In other words, $f_{2}$ has arity 2 , just like $f_{1}$, because it also requires two arguments before doing serious work. The function $f_{3}$ has the same type as $f_{1}$, but it must take its arguments one at a time: in the call $\left(\operatorname{map}\left(f_{3} 100\right) x s\right)$ we expect (expensive 100) to be computed at most once, whereas the same call for $f_{1}$ would recompute (expensive 100) for each element of $x s$. Similarly, $f_{4}$ has arity 1 : it cannot be $\eta$-expanded without the risk of computing (expensive 100) repeatedly.

These choices become particularly clear in a call-by-value language with side effects. For example, if (expensive 100) printed something on the screen, the fact that it is evaluated only once-rather than once for each element of $x s$-is a matter of semantics, not mere efficiency. Even in a pure language like Haskell, an optimizing compiler should still treat computation as a sort of effect; it must, for example, avoid changing the asymptotic efficiency of the program.

In light of these examples, here are two informal definitions of arity:

- An expression $e$ has arity $n$ when it can be soundly $\eta$-expanded to $\left(\lambda x_{1} \ldots x_{n}\right.$.e $\left.x_{1} \ldots x_{n}\right)$. "Soundness" concerns semantics in an effectful language, but "only" efficiency in a pure one.

- An expression $e$ has arity $n$ when it does no "useful work" until it is applied to $n$ arguments; hence those arguments can be passed simultaneously.

Since the type of a function does not describe its arity (compare $f_{1}$ and $f_{3}$ above), practical compilers like GHC perform arity analysis based on intensional properties: the form of the expression determines its arity. In its simplest form, we can just count $\lambda$ s. Since that is pessimistic on examples like $f_{2}$, GHC uses a variety of simple static heuristic analyses [e.g., Breitner 2014]. The focus of this paper is not on arity analysis, however, but rather on the intermediate language in which we can memorialise the results of that analysis.

\subsection{Arity in the Intermediate Language}

However arity analysis is done, we need a way to express its results in the intermediate language. In GHC, this is done through an informal decoration on each binder describing its arity. ${ }^{1}$ This turns out to be extremely unsatisfactory in practice: GHC has lots and lots of dark corners as a result of this rather squishy notion of arity.

It would be much better if arity were a solid, statically-checked part of the intermediate language. How is that possible? In the world of $\lambda$-calculi, we are familiar with calculi having unrestricted $\beta$ and $\eta$ rules (such as the call-by-name $\lambda$-calculus), as well as calculi having restricted $\beta$ and $\eta$ (such as the call-by-value [Sabry and Wadler 1997] or call-by-need $\lambda$-calculus [Ariola and Felleisen 1997]). The latter are often used as intermediate languages in compilers, to avoid recomputation of, say, integers. The exact restrictions depend on the language being compiled: its evaluation strategy, whether there are side effects, and so on. Our key idea, introduced by Downen et al. [2019], is this:

Define an intermediate language $(\mathcal{I} \mathcal{L})$ that has unrestricted $\eta$ expansion for functions, while allowing for restricted $\beta$ reduction on other types of expressions.

This is an unusual choice, so we use a different notation for functions, $\lambda(x: \tau) . e: \tau \leadsto \sigma$, where the $(\sim)$ arrow denotes the new primitive function type. Now arity can be read from types: you can freely $\eta$-expand any expression $e: \tau \leadsto \sigma$, without reference to the form of $e$. The previous paper [Downen et al. 2019] and this one are simply working out the consequences of this one idea.

\subsection{Currying}

If the primitive function type of our intermediate language allows unrestricted $\eta$-expansion, how can we express functions like $f_{3}$ that intentionally use currying as a way to avoid work duplication?

\footnotetext{
${ }^{1}$ For aficionados, it is part of the binder's IdInfo
} 
We can do so like this:

$$
\begin{aligned}
& f_{3}: \text { Int } \leadsto\{\text { Int } \leadsto \text { Int }\} \\
& f_{3}=\lambda x . \text { let } z=\text { expensive } x \text { in } \operatorname{Clos}(\lambda y \cdot y+z)
\end{aligned}
$$

The type Int $\leadsto\{$ Int $\leadsto$ Int $\}$ makes explicit that $f_{3}$ is an arity-1 function that returns a closure, denoted by the curly braces, inside which is another arity- 1 function. In the term language, $\lambda$ builds a primitive function of type $\tau \leadsto \sigma$, while Clos builds a closure of type $\{\tau\}$. Here are the (slightly simplified) introduction (-I) and elimination (-E) rules:

$$
\begin{array}{cc}
\frac{\Gamma, x: \tau \vdash e: \sigma}{\Gamma \vdash \lambda x: \tau . e: \tau \leadsto \sigma} \text { Fun-I } & \frac{\Gamma \vdash e: \tau}{\Gamma \vdash \operatorname{Clos} e:\{\tau\}} \text { CLO-I } \\
\frac{\Gamma \vdash e: \tau \leadsto \sigma \quad \Gamma \vdash e^{\prime}: \tau}{\Gamma \vdash e e^{\prime}: \sigma} \text { FUn-E } & \frac{\Gamma \vdash e:\{\tau\}}{\Gamma \vdash \operatorname{App} e: \tau} \text { CLO-E }
\end{array}
$$

The App form unboxes the primitive function wrapped up by Clos.

Our plan, then, is to desugar the source-language function type $\tau \rightarrow \sigma$ into the intermediatelanguage type as $\{\tau \leadsto \sigma\}$, adding the corresponding Clos and App constructs in the terms. ${ }^{2}$ Then we can perform arity analysis, and express its results by transforming the intermediate language program into one with fewer intermediate Clos nodes.

\subsection{Functions Are Called, Not Evaluated}

If we are able to freely $\eta$-expand, we must only evaluate functions when they are called. Consider:

$$
x=\text { let } f: \text { Int } \leadsto \text { Int }=\text { expensive } 100 \text { in } \ldots f \ldots f \ldots
$$

When might expensive 100 be evaluated? In a strict language, it is evaluated right away, so the value (some first-class function) can be bound to $f$ before continuing with the body of the let. In a lazy language like Haskell, expensive 100 might be forced long before $f$ needs to be called, as in seq $f y$ or with a strict pattern. But in both cases, evaluating $f$ without calling it violates the unrestricted $\eta$ we desire. After all, the definition of $x$ is $\eta$-equivalent to:

$$
x^{\prime}=\text { let } f: \text { Int } \leadsto \text { Int }=\lambda \text { y.expensive } 100 y \text { in } \ldots f \ldots f \ldots
$$

After $\eta$-expansion, the only way to evaluate expensive 100 is to call $f$ with an argument. Unrestricted $\eta$ means that $x$ and $x^{\prime}$ must be the same-in both semantics and asymptotic cost.

Unrestricted $\eta$-expansion requires a matching evaluation order for function bindings; the evaluator must treat every expression $e: \tau \leadsto \sigma$ the same as $(\lambda x$.e $x)$. Yet, we do not want to change the evaluation order for expressions of other types, like Int. Thus, our language's semantics becomes type-directed: it is only the type of $f$ (in this case, Int $\sim$ Int) that tells us not to evaluate the right-hand side. In a precise sense (spelled out formally in Section 4), we cannot evaluate functions (e.g., due to strictness, seq, etc.); functions can only be called. Furthermore, we will see in Section 3.7 that functions are, in fact, $\eta$-expanded during compilation to a lower level. So a semantics that prematurely evaluates functions before they are called is not supported by our machine language.

\section{WHY KINDS ARE CALLING CONVENTIONS}

So far we have reviewed the ideas of Downen et al. [2019]. We are now ready to introduce the problem we tackle in this paper, and our solution to it.

Previous research asserted types are calling conventions [Bolingbroke and Peyton Jones 2009]. We respectfully disagree, instead claiming that kinds are calling conventions. As we shall see, this principle offers a unified framework combining several previous works on representations

\footnotetext{
${ }^{2}$ In Downen et al. [2019], the intermediate language itself had $t$ wo arrows, $(\rightarrow)$ as well as $(\leadsto)$, but we have found it clearer to have only one arrow for primitive functions plus the closure type $\{\tau\}$.
} 
[Eisenberg and Peyton Jones 2017; Peyton Jones and Launchbury 1991], arity [Bolingbroke and Peyton Jones 2009; Downen et al. 2019; Marlow and Peyton Jones 2004], and mixed evaluation strategies [Downen and Ariola 2018] in intermediate languages. Specifically, we provide a type system in which the kind $\kappa$ of a type $\tau: \kappa$ classifies all the intensional properties needed to compile an expression of type $\tau$, namely its representation, arity, and levity.

\subsection{Why Polymorphism Is a Problem}

Consider this example of a polymorphic definition, in our intermediate language

$$
\begin{aligned}
& \text { poly }: \forall a .(\text { Int } \leadsto \text { Int } \leadsto a) \sim(a, a) \\
& \text { poly }=\Lambda a . \lambda(f: \text { Int } \leadsto \text { Int } \leadsto a) \text {. let } g: \text { Int } \leadsto a=f 3 \text { in }(g 5, g 4)
\end{aligned}
$$

What is the arity of $f$ and $g$ ? With "arities in the types," we count the arrows, and answer 2 and 1 respectively. But what if a were instantiated by Bool $\leadsto$ Bool? Then suddenly the answers become 3 and 2 respectively. Yikes!

One solution is to monomorphize the entire program, creating type-specialized versions of each polymorphic function. This is patently unsatisfactory. First, it is a whole-program transformation. Second, some languages (including Haskell and OCaml) support polymorphic recursion, which makes static monomorphization impossible. Third, there is a risk of creating many copies of essentially the same function, many of which wastefully compile to identical machine code.

Instead, we retain the traditional type-erasure model: each polymorphic function is compiled to a single chunk of machine code that works the same no matter how its type is specialized. Under this model, our function poly above is problematic. To compile code we must know the arity of every function we call (because its arity determines its calling convention, and thus, what code to generate), but in poly we do not know a stable arity of $f$ or $g$ for every instance of $a$.

\subsection{Nonuniform Representations and Polymorphism}

Interestingly, this exact same problem has arisen before, in the context of unboxed data types. A contribution of this paper is to show that both can be solved with the same idea.

Nearly thirty years ago, GHC introduced the idea of distinguishing boxed and unboxed data types in its intermediate language [Peyton Jones and Launchbury 1991]. They introduced two distinct types for integers: Int\# for primitive, unboxed machine integers; and Int for boxed integers, represented as a pointer to a heap-allocated object and defined as:

$$
\text { data Int }=\mathrm{I} \# \text { Int\# }
$$

The (sole) goal of distinguishing these two types is efficiency. If we had only boxed integers, then even simple addition would be forced to evaluate and unbox each argument, then box up the result. By making boxing and unboxing explicit we expose much more low-level information to the optimizer, and can eliminate lots of intermediate boxes. For example, assume we have plus\# and minus\# primitive operations, both of type Int\# $\rightarrow$ Int $\rightarrow$ Int $\#$, and consider:

$$
\begin{array}{lll}
\text { plus, minus }: \text { Int } \rightarrow \text { Int } \rightarrow \text { Int } & \text { sumFrom } & \text { : Int } \rightarrow \text { Int } \\
\text { plus }(\mathrm{I} \# x)(\mathrm{I} \# y)=\mathrm{I} \#(\text { plus\# } x y) & \text { sumFrom }(\mathrm{I} \# 0)=\mathrm{I} \# 0 \\
\text { minus }(\mathrm{I} \# x)(\mathrm{I} \# y)=\mathrm{I} \#(\text { minus\# } x y) & \text { sumFrom } x=\operatorname{plusx}(\operatorname{sumFrom}(\text { minus } x(\mathrm{I} \# 1))
\end{array}
$$

This definition is wasteful; each recursive step allocates several new boxes on the heap only to be immediately used by plus, minus, and sumFrom. Instead, as Peyton Jones and Launchbury [1991] show, the recursive function can be optimized using the worker/wrapper transformation, like so:

sumFrom $\quad:$ Int $\rightarrow$ Int
sumFrom $(\mathrm{I} \# x)=\mathrm{I} \#($ sumFrom\# $x)$

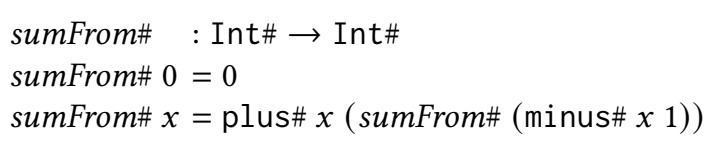


Now, the recursion is done by the more efficient sumFrom\# function which works directly on machine integers; no boxes are allocated or consumed, and so sumFrom\# can be compiled with no intermediate allocations. sumFrom becomes a wrapper around sumFrom\#, just handling the issues of boxing and unboxing. This is a huge gain in both time and space.

Notice the similarities between this work on unboxed data types and the earlier discussion about arity. In both cases, we make $\mathcal{I} \mathcal{L}$ expose more primitive, but less convenient, types of values (primitive functions and unboxed integers respectively), along with a way to explicitly "box" them into a more convenient form (using Clos and I\# respectively) and later "unbox" them (using App and pattern matching on I\# respectively). Making these boxing and unboxing operations explicit in the syntax of $\mathcal{I} \mathcal{L}$ programs makes them accessible to the optimizer.

Alas, unboxed types cause trouble with polymorphism. For example, consider the generic, higherorder binary application function:

$$
\text { binapp : } \forall a b c .(a \rightarrow b \rightarrow c) \rightarrow a \rightarrow b \rightarrow c \quad \text { binapp } f x y=f x y
$$

To compile binapp to a single piece of code, we cannot expect the calls binapp plus and binapp plus\# to both work; binapp would somehow have to handle arguments $x$ and $y$ with different representations (perhaps stored in different registers) to pass them along to plus versus plus\#.

Notice that this is the exact same problem that we had with poly in Section 3.1: the code we compile for binapp depends on how the type variables $a$ and $b$ are instantiated.

\subsection{A Stop-Gap Solution}

Because of the difficulty with polymorphism, unboxed types were originally introduced with a draconian restriction on polymorphism: polymorphic type variables (like a, b, $c$ in binapp) cannot be instantiated with unboxed types (like Int\#) [Peyton Jones and Launchbury 1991]. The mechanism for enforcing the restriction was the kind system: Int has kind $\star$ (the kind of ordinary data types), but Int\# has a different kind \# (the kind of unboxed data). GHC then required that the kind of a quantified type variable $t$ could be $\star$ or, say, $(\star \rightarrow \star)$, but never $\#$.

Since this is the same problem as the one we encountered for arity, in function poly in Section 3.1, we might expect the same solution to work. And indeed that is the approach adopted by Downen et al. [2019]: type variables cannot range over arrow types $\tau \leadsto \sigma$, whose kind is different from $\star$.

While this approach has served GHC well for nearly three decades, it has two major inadequacies:

(1) Too restrictive. Consider the error function, which prints a message and prematurely ends the program. It can have the type $\forall t$.String $\rightarrow t$. Because error never returns a value, it doesn't matter how $t$ is represented; truly the same code can be used no matter if $t$ is boxed or unboxed. But the draconian restriction on unboxed types rejects this kind of polymorphism. Instead, specialized versions are needed, like errorInt\# : String $\rightarrow$ Int\#, even though the compiled code is identical. For error we would prefer to be able to say that the representation of $t$ doesn't matter.

(2) Kind polymorphism. In a language with kind polymorphism, we can write a term with type $\forall k . \forall(a: k) . \tau$. Here, $a$ can have any kind $k$, including \#. We have now lost our ability to prevent quantifiers over \#-types, and need a new solution to the problem of uncompilable polymorphism. ${ }^{3}$

\footnotetext{
${ }^{3}$ A particularly knowledgeable reader might be aware that GHC supported kind polymorphism and restricted quantification for a few years. This worked because a type like $\forall k(a: k) . a \rightarrow a$ is ill-kinded; the $\rightarrow$ type former puts requirements on $a$. However, with kind equalities [Weirich et al. 2013], a type variable $a: k$ can be cast to a more specific kind, causing chaos. In fact, this interaction originally spurred the development of Eisenberg and Peyton Jones [2017].
} 


\subsection{A Better Way: Look to the Kinds}

Fortunately, both (1) and (2) can be solved, by using... more polymorphism. Let's start with representation polymorphism, using the approach of Eisenberg and Peyton Jones [2017]. We give Int and Int\# these more refined kinds:

\section{Int : TYPE PtrR Int\# : TYPE IntR}

where TYPE : Rep $\rightarrow \star$, and PtrR, IntR : Rep. ${ }^{5}$ The idea is that, given $\tau:$ TYPE $r$, values of type $\tau$ have a runtime representation described by $r$. Now we can quantify over types represented by a heap pointer with $\forall(a$ : TYPE PtrR). $\tau$. But we can also define representation-polymorphic functions-like error $: \forall(r: \operatorname{Rep})(a:$ TYPE $r)$. String $\rightarrow a$-solving problem (1).

What of problem (2)? Instead of limiting how type variables can be instantiated (which is incompatible with full kind polymorphism), we instead add side conditions to the typing rules for abstraction and application, excluding uncompilable programs like so:

$$
\frac{\Gamma, x: \tau \vdash e: \sigma \quad \Gamma \vdash \tau \text { mono-rep }}{\Gamma \vdash \lambda x: \tau . e: \tau \leadsto \sigma} \text { Fun-I } \quad \frac{\Gamma \vdash e: \tau \leadsto \sigma \quad \Gamma \vdash e^{\prime}: \tau \quad \Gamma \vdash \tau \text { mono-rep }}{\Gamma \vdash e e^{\prime}: \sigma} \text { Fun-E }
$$

The $\tau$ mono-rep caveat says $\tau$ 's kind must be representation-monomorphic; that is, it can be TYPE PtrR; or TYPE IntR; but not TYPE $r$. For example, we might try to give binapp this type

$$
\begin{aligned}
& \text { binapp : } \forall\left(r_{a}, r_{b}, r_{c}: \operatorname{Rep}\right)\left(a: \operatorname{TYPE} r_{a}\right)\left(b: \operatorname{TYPE} r_{b}\right)\left(c: \operatorname{TYPE} r_{c}\right) .(a \rightarrow b \rightarrow c) \rightarrow a \rightarrow b \rightarrow c \\
& \text { binapp } f x y=f x y
\end{aligned}
$$

But that should be rejected because we cannot compile the call ( $f x y)$ without knowing how $x$ and $y$ are represented (and thus, how to retrieve them and pass them to $f$ ). Indeed, it is rejected (by both Fun-I and Fun-E). On the other hand, perhaps surprisingly, this type for binapp is fine:

$$
\text { binapp : } \forall\left(r_{c}: \text { Rep }\right)(a: \text { TYPE PtrR })(b: \operatorname{TYPEPtrR})\left(c: \operatorname{TYPE} r_{c}\right) .(a \rightarrow b \rightarrow c) \rightarrow a \rightarrow b \rightarrow c
$$

Notice that binapp can be representation-polymorphic in the return type $c$. Because our compiler supports tail-call elimination, $f$ is the one to return a value of type $c$ to the caller, not binapp.

\subsection{Arity Polymorphism}

Arity and representations share problems (1) and (2), and thankfully, they share solutions, too. We add a second parameter to TYPE that describes the arity of the function, like this: ${ }^{6}$

$$
\begin{aligned}
& \text { poly }: \forall(a: \text { TYPE PtrR Call[2]). (Int } \leadsto \text { Int } \leadsto a) \leadsto(a, a) \\
& \text { poly }=\Lambda(a: \text { TYPE PtrR Call[2]). } \lambda(f: \text { Int } \leadsto \text { Int } \leadsto a) \text {. let } g: \text { Int } \leadsto a=f 3 \text { in }(g 5, g 4)
\end{aligned}
$$

Here, $a$ 's kind says that it only ranges over arity-2 types, of kind TYPE PtrR Call[2], while $f$ 's type Int $\leadsto$ Int $\leadsto a$ has kind TYPE PtrR Call[4], hence $f$ has arity 4 in total. Similarly, $g:$ Int $\leadsto a$ of kind TYPE PtrR Call[3] has arity 3. In short, the calling convention of a function (how many arguments to pass simultaneously) is described by its kind. Of course, this meant that we had to choose a particular arity for $a$, just as we had to choose a particular representation for the $a$ and $b$ in binapp's type. We enforce this "particular arity" constraint not at the point of abstraction, but at the point of application. Here is the augmented Fun-E rule:

$$
\begin{array}{llll}
\Gamma \vdash e: \tau \leadsto \sigma \quad \Gamma \vdash e^{\prime}: \tau & \Gamma \vdash \tau \text { mono-rep } & \Gamma \vdash \tau \text { mono-conv } \\
& \Gamma \vdash e e^{\prime}: \sigma & \text { Fun-E }
\end{array}
$$

where the new side condition $\tau$ mono-conv ensures TYPE's second parameter is statically known. Preparing an argument of function type is precisely the point at which a compiler must compile

\footnotetext{
${ }^{4}$ The paper Levity Polymorphism in fact describes representation polymorphism. For levity polymorphism, see Section 3.6.

${ }^{5} \mathrm{GHC}$ classifies representations like PtrR, IntR, etc., as RuntimeRep; here, we shorten the name to just Rep.

${ }^{6}$ These kinds are somewhat simplified; the full story is in Section 4.
} 
code for that argument that actually takes the number of arguments specified by its kind. Requiring the calling convention be monomorphic fixes that number statically; it can't be a type variable, say.

\subsection{Evaluation Strategy and Levity}

We have discussed arity and representation, two of our three design axes. But what of levity?

When compiling a function call $f(1+1)$, we must know the evaluation strategy to use: do we evaluate $(1+1)$ before calling $f$ or after, on-demand? Programming languages typically commit to a choice here, which is usually to evaluate $(1+1)$ before the function call-the eager, call-byvalue strategy. Haskell makes the opposite choice, implementing the lazy, call-by-need strategy; $(1+1)$ is evaluated only when its value is needed, and only once. But regardless of a language's choice of evaluation strategy, some scenarios require the opposite. Programmers in eager languages sometimes use constructs like Delay and Force [Wadler et al. 1998] to embed lazy evaluation, and programmers in lazy languages manually introduce strictness (such as Haskell's seq) to force eager evaluation. So a compiler's intermediate language should support both lazy and eager evaluation, and ideally without favoring either over the other.

We can use types (or, more precisely, their kinds) to control evaluation order. For example, suppose we have types In $\mathrm{L}^{\mathrm{L}}$ and $I n \mathrm{t}^{\mathrm{U}}$, where the $\mathrm{L}$ stands for "Lifted" (the type has an extra bottom element, $\perp$, denoting divergent computation) and U for "Unlifted" (the type has no extra bottom element). Operationally, a value of lifted type must be represented as a pointer to a heap-allocated object because it may be an unevaluated thunk; a value of unlifted type may well still be represented by a pointer, but to the value itself, not a thunk. Variables of unlifted types cannot be bound to (divergent) computations, so they must be evaluated eagerly at binding-time; variables of lifted types may be bound to any unevaluated computation, so those bindings may be evaluated lazily. The types of an eager language (like OCaml) are all unlifted, whereas the types of a lazy language (like Haskell) are all lifted. But our $\mathcal{I} \mathcal{L}$ supports both, and hence can be a target for both.

Once again, we can track levity in the kinds, like this:

$$
\text { Int }^{\mathrm{L}} \text { : TYPE PtrR Eval }{ }^{\mathrm{L}} \quad \text { Int }^{\mathrm{U}} \text { : TYPE PtrR Eval }^{\mathrm{U}} \quad \text { Int\# : TYPE IntR Eval }{ }^{\mathrm{U}}
$$

The levity of a type is all about its evaluation strategy. We already know primitive functions cannot be evaluated without calling them (Section 2.4), and thus a type has either a levity or an arity. We accordingly re-use the second component of the kind TYPE, which in Section 3.5 described the arity of a primitive function, using $\mathrm{Eval}^{\mathrm{L}}$ and $\mathrm{Eval}^{\mathrm{U}}$ to classify lifted and unlifted data types respectively. Reflecting this dual use, we describe the second argument of TYPE as the kind's convention, connoting "calling convention" for functions and "evaluation convention" for data.

Once again, we can be polymorphic in both levity (Section 4.4) and convention (Section 4.5). And we still keep the program compilable despite type erasure using suitable side conditions (Section 4.6). In the case of levity, the mono-conv premise in Fun-E (recall Section 3.5) is exactly what is needed to specify whether to compile this application using call-by-need or call-by-value.

\subsection{From $\mathcal{I L}$ to $\mathcal{M L}$}

Returning to arities, how can poly in Section 3.5 be compiled? In particular, since $g$ 's kind specifies that it has arity 3, we must compile $g$ to code that takes three arguments. So, before generating code, we $\eta$-expand $g$. But apparently we can't, at least not in the confines of $\mathcal{I} \mathcal{L}$ 's type system, because $a$ is not an arrow type!

To have any hope of solving this problem, we must do more than say $a$ is an arity-2 type. We must also spell out the representations of its two arguments, so that we can generate code for passing the $\eta$-expanded function arguments. In poly, the explicated calling convention looks like:

$$
\begin{aligned}
\text { poly }= & \Lambda(a: \text { TYPE PtrR Call[PtrR, IntR }]) . \lambda(f: \text { Int } \leadsto \text { Int } \leadsto a) . \\
& \text { let } g: \text { Int } \leadsto a=f 3 \text { in }(g 5, g 4)
\end{aligned}
$$




$$
\begin{aligned}
& \text { Kind } \ni \quad \kappa::=\text { TYPE } \rho v \\
& \text { Type } \ni \tau, \sigma::=t\left|T_{p}\right| T_{d}|\tau \leadsto \sigma|{ }^{\gamma}\{\tau\} \mid \forall \chi . \sigma \quad \text { TyVar } \ni \quad \chi::=t: \kappa|r| g \mid n \\
& \text { Representation } \ni \quad \rho::=r|\operatorname{PtrR}| \operatorname{IntR} \mid \ldots \quad \text { PrimType } \ni T_{p}::=\operatorname{Int} \# \mid \ldots \\
& \text { Levity } \ni \quad \gamma::=g|\mathrm{~L}| \mathrm{U} \quad \text { DataType } \ni T_{d}::=\operatorname{Int}^{\gamma} \mid \ldots \\
& \text { Convention } \ni \quad v::=n\left|\mathrm{Eval}^{\gamma}\right| \mathrm{Call}[\alpha] \quad \text { Arity } \ni \alpha::=\rho, \alpha|\varepsilon| \operatorname{arity}(v) \\
& \text { Expr } \ni e::=x|c| \mathrm{I}^{\gamma} e \mid \text { case } e \text { of I\# } x \rightarrow e^{\prime}|\lambda x: \tau . e| e e^{\prime}\left|\mathrm{Clos}^{\gamma} e\right| \operatorname{App} e|\Lambda \chi . e| e \phi \\
& \text { Const } \ni c::=i \mid \text { op } \quad \text { PrimOp } \ni \text { op }::=\text { error } \mid \ldots \\
& \text { Answer } \ni A::=x|c| \mathrm{I}^{\gamma} A\left|\mathrm{Clos}^{\gamma} e\right| \Lambda \chi \cdot A \mid A \phi \quad \text { Erasable } \ni \phi::=\tau|\rho| \gamma \mid v
\end{aligned}
$$

Fig. 1. Syntax of $\mathcal{I L}$ : An intermediate language with levity, representation, and arity polymorphism

The Call[PtrR, IntR] in $a$ 's kind describes the representation of its two arguments.

Before code generation, we compile from $\mathcal{I} \mathcal{L}$ into a lower-level representation $\mathcal{M L}$ (suggesting "machine language"). $\mathcal{M L}$ is still statically typed, but much more coarsely: $\mathcal{M L}$ 's types correspond to representations and calling conventions. So compiled polymorphic code might look like:

$$
\begin{aligned}
& \text { poly }=\lambda(f: \mathrm{PtrR}) . \text { let } g: \mathrm{PtrR}=\lambda(x: \mathrm{PtrR}, y: \mathrm{PtrR}, z: \text { IntR }) . f(3, x, y, z) \\
& \text { in }(\lambda(y: \operatorname{PtrR}, z: \text { IntR }) . g(5, y, z), \quad \lambda(y: P \operatorname{trR}, z: \operatorname{IntR}) \cdot g(4, y, z))
\end{aligned}
$$

In $\mathcal{M L}$ the functions are uncurried, and specify all their arguments and their representations. As such, every function is fully $\eta$-expanded, and every call is fully saturated. This representation is less convenient for the optimizer (for which we use $\mathcal{I L}$ ), but is just right for the code generator. We discuss $\mathcal{M L}$, and the lowering transformation from $\mathcal{I} \mathcal{L}$ to $\mathcal{M L}$, in Section 6 .

\section{THE INTERMEDIATE LANGUAGE $(\mathcal{I} \mathcal{L})$}

Our intermediate language, which we call $\mathcal{I} \mathcal{L}$, is an explicitly-typed $\lambda$-calculus based closely on System F, with its syntax (Fig. 1), formation of types (Fig. 2), and type system (Fig. 3). We cover the kind system of $\mathcal{I} \mathcal{L}$ in more detail in Sections 4.2 to 4.7.

The syntax for expressions $e$ is given in Fig. 1, and includes the following forms.

- Variables $x$, and constants $c$.

- Primitive integers have type Int\# : TYPE IntR Eval ${ }^{U}$, and are represented by a machine integer (hence IntR in the kind). There are numeric constants, $i:$ Int\#, and primitive functions (op) that operate over Int\#, such as plus\# : Int\# $\leadsto$ Int\# $\leadsto$ Int\#.

- Boxed integers have type Int ${ }^{\gamma}$ : TYPE PtrR Eval ${ }^{\gamma}$, where the levity $\gamma$ can be $\mathrm{L}, \mathrm{U}$ or a levity variable $g$. A data-constructor application $\mathrm{I}^{\gamma} e: \mathrm{Int}^{\gamma}$ allocates a box containing the value of $e$ (see Section 3.6). Such I\# boxes are unpacked by a pattern-matching case.

- Primitive functions are introduced and eliminated with the familiar forms $\lambda x: \tau . e$ and $e e^{\prime}$. As discussed in Section 2.2 we use a wavy arrow $(\tau \leadsto \sigma)$ to remind ourselves that in this $\lambda$-calculus functions have some unusual behavior: they enjoy unrestricted $\eta$-expansion. Multiple arguments, even though they are curried, are always passed simultaneously; e.g., if $f:$ Int\# $\leadsto$ Int\# $\leadsto$ Int\# $\leadsto$ Int\#, then $f$ is always called with all three arguments.

- Function closures are introduced and eliminated with the forms $\mathrm{Clos}^{\gamma} e$ and App e, respectively. Similar to boxed integers, $\mathrm{Clos}^{\gamma} e$ allocates a new closure containing $e$, where $\gamma$ records the levity of the computation responsible for that allocation, just as with $\mathrm{I} \#^{\gamma}$.

- Type abstraction $\Lambda \chi$.e and application e $\phi$, have the same meaning as in System F. While their operational interpretation in $\mathcal{I} \mathcal{L}$ is analogous to $\lambda x: \tau$.e and $e e^{\prime}$, these abstractions and applications are fully erased by compilation to a lower-level representation. The only new 
Formation rules for kinds, levities, representations, conventions, arities:

$$
\begin{aligned}
& \frac{\Gamma \vdash \rho \text { rep } \Gamma \vdash v \text { conv }}{\Gamma \vdash \operatorname{TYPE} \rho v \text { kind }} \\
& \Gamma \vdash \kappa \text { kind } \Gamma \vdash \operatorname{TYPE} \rho v \text { kind }
\end{aligned}
$$

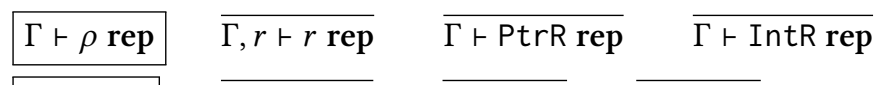

$$
\begin{aligned}
& \Gamma \vdash \gamma \text { lev } \overline{\Gamma, g \vdash g \text { lev }} \overline{\Gamma \vdash L \text { lev }} \overline{\Gamma \vdash U \text { lev }} \\
& \Gamma \vdash v \operatorname{conv} \quad \frac{\Gamma \vdash \gamma \text { lev }}{\Gamma, n \vdash n \operatorname{conv}} \quad \frac{\Gamma \vdash \alpha \text { ari }}{\Gamma \vdash \mathrm{Eval}^{\gamma} \operatorname{conv}} \quad \frac{\Gamma+\operatorname{Call}[\alpha] \operatorname{conv}}{\Gamma \vdash \rho \operatorname{rep}}
\end{aligned}
$$

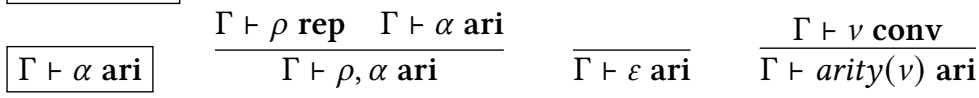

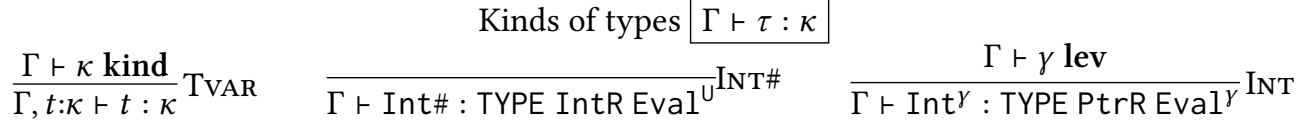

$$
\begin{aligned}
& \frac{\Gamma \vdash \tau_{1}: \operatorname{TYPE} \rho_{1} v_{1} \quad \Gamma \vdash \tau_{2}: \operatorname{TYPE} \rho_{2} v_{2}}{\Gamma \vdash \tau_{1} \leadsto \tau_{2}: \operatorname{TYPE} \operatorname{PtrR} \operatorname{Call}\left[\rho_{1}, \operatorname{arity}\left(v_{2}\right)\right]} \text { FUN } \quad \frac{\Gamma \vdash \gamma \text { lev } \quad \Gamma \vdash \tau: \operatorname{TYPE} \rho v}{\Gamma \vdash \gamma\{\tau\}: \operatorname{TYPE} \operatorname{PtrR} \operatorname{Eval}^{\gamma}} \text { CLO } \\
& \frac{\Gamma, \chi \vdash \sigma: \operatorname{TYPE} \rho v \quad \Gamma \vdash \operatorname{TYPE} \rho v \text { kind }}{\Gamma \vdash \forall \chi \cdot \sigma: \operatorname{TYPE} \rho v} \text { ForALL } \frac{\Gamma \vdash \tau: \kappa \quad \kappa=\kappa^{\prime}}{\Gamma \vdash \tau: \kappa^{\prime}} \text { K-CONV }
\end{aligned}
$$

Reflexivity, transitivity, symmetry, and compatibility for $\kappa=\kappa^{\prime}$, plus the following rules:

$$
\begin{aligned}
& \operatorname{arity}\left(\mathrm{Eval}^{\gamma}\right)=\varepsilon \quad \operatorname{arity}(\mathrm{Cal}[\alpha])=\alpha \\
& \text { Monomorphism restrictions: } \\
& \frac{\Gamma \vdash \tau: \text { TYPE } \rho v+\rho \text { rep }}{\Gamma \vdash \tau \text { mono-rep }} \text { MONO-REP } \frac{\Gamma \vdash \tau: \text { TYPE } \rho v \vdash v \text { conv }}{\Gamma \vdash \tau \text { mono-conv }} \text { MONO-CONV }
\end{aligned}
$$

Fig. 2. Kind and levity system of $\mathcal{I} \mathcal{L}$

addition here is that binders $\chi$ and arguments $\phi$ range over four different sorts of type-level variables and arguments (all erasable). The expressiveness and restrictions on these different kinds of polymorphism are detailed in Sections 4.3 to 4.8. Type binders are annotated with their kinds, and other binders are distinguished by their naming convention; we use a Latin name for type-level variables and the corresponding Greek name for the syntactic category. For clarity, we sometimes state the sort of variable being quantified ( $r$ :Rep, $g$ :Lev, or $n$ :Conv).

A subset of these expressions are Answers (denoted by A, Fig. 1), meaning that they are possible results of evaluation. This classification accounts for the eventual type erasure mentioned above. The apparent redex $(\Lambda \chi . A) \phi$ is an answer, because it is erased to $A$ at compile-time. Answers include closures $\mathrm{Clos}^{\gamma} e$ as usual, but not primitive functions $\lambda x: \tau . e$; recall that functions are called instead of evaluated, and thus cannot be answers of evaluation (Section 2.4).

The rules for well-formed types and kinds are given in Fig. 2, and include type variables $t$, primitive types $T_{p}$ (of which we supply one, Int\#), algebraic data types $T_{d}$ (of which we supply one, Int), polymorphic types $\forall \chi . \sigma$, primitive function types $\tau \leadsto \sigma$ and a closure type ${ }^{\gamma}\{\tau\}$.

$\mathcal{I} \mathcal{L}$ 's type system given in Fig. 3 ensures the following guarantees for well-behaved programs:

- Static Compilation: Every well-typed program can be compiled to a lower-level representation for a monomorphic machine, which models details such as specialized registers (for integers versus pointers) and function calls with multiple arguments (Theorem 3).

- Type Safety: If a well-typed program is equal to a number (as per Section 4.9), then its compiled code computes that number (Theorem 4). In particular, executing well-typed programs never 


$$
\begin{aligned}
& \text { Types of expressions } \Gamma \vdash e: \tau \\
& \frac{\Gamma \vdash \tau: \kappa}{\Gamma, x: \tau \vdash x: \tau} \operatorname{VAR} \quad \frac{c: \tau}{\Gamma \vdash c: \tau} \text { CONST } \\
& \frac{\Gamma \vdash e: \text { Int\# }}{\Gamma \vdash \mathrm{I} \# \gamma e: \operatorname{Int}^{\gamma}} \text { InT-I } \frac{\Gamma \vdash e: \operatorname{Int}^{\gamma} \quad \Gamma, x: \text { Int\# } \vdash e^{\prime}: \tau}{\Gamma \vdash \operatorname{case} e \text { of } \mathrm{I} \# x \rightarrow e^{\prime}: \tau} \text { INT-E } \\
& \frac{\Gamma, x: \tau \vdash e: \sigma \quad \Gamma \vdash \tau \text { mono-rep }}{\Gamma \vdash \lambda x: \tau . e: \tau \leadsto \sigma} \text { FUn-I } \frac{\Gamma \vdash e: \tau \leadsto \sigma \quad \Gamma \vdash A: \tau \quad \Gamma \vdash \tau \text { mono-rep }}{\Gamma \vdash e A: \sigma} \text { Fun-A-E } \\
& \frac{\Gamma \vdash e: \tau \leadsto \sigma \quad \Gamma \vdash e^{\prime}: \tau \quad \Gamma \vdash \tau \text { mono-rep } \quad \Gamma \vdash \tau \text { mono-conv }}{\Gamma \vdash e e^{\prime}: \sigma} \text { Fun-E } \\
& \frac{\Gamma \vdash e: \tau \quad \Gamma \vdash \tau \text { mono-conv }}{\Gamma \vdash \operatorname{Clos}^{\gamma} e:{ }^{\gamma}\{\tau\}} \text { CLO-I } \quad \frac{\Gamma \vdash e:{ }^{\gamma}\{\tau\}}{\Gamma \vdash \operatorname{App} e: \tau} \text { CLO-E } \\
& \frac{\Gamma, \chi \vdash e: \sigma \quad \Gamma \vdash \forall \chi \cdot \sigma: \kappa}{\Gamma \vdash \Lambda \chi \cdot e: \forall \chi \cdot \sigma} \forall-\mathrm{I} \quad \frac{\Gamma \vdash e: \forall \chi \cdot \sigma \quad \Gamma \vdash[\phi / \chi] \text { poly }}{\Gamma \vdash e \phi: \sigma[\phi / \chi]} \forall-\mathrm{E} \\
& \text { Polymorphic instantiations: } \Gamma \vdash[\phi / \chi] \text { poly } \\
& \frac{\Gamma \vdash \gamma \text { lev }}{\Gamma \vdash[\gamma / g] \text { poly }} \quad \frac{\Gamma \vdash \rho \text { rep }}{\Gamma \vdash[\rho / r] \text { poly }} \quad \frac{\Gamma \vdash v \text { conv }}{\Gamma \vdash[v / n] \text { poly }} \quad \frac{\Gamma \vdash \tau: \kappa \Gamma \vdash \kappa \text { kind }}{\Gamma \vdash[\tau / t: \kappa] \text { poly }} \\
& \text { Types of constants: } c: \tau \\
& i: \text { Int\# error }: \forall r . \forall g . \forall\left(t: \text { TYPE } r \mathrm{Eval}^{g}\right) \text {. Int\# } \leadsto t
\end{aligned}
$$

Fig. 3. Type system of $\mathcal{I L}$

gets stuck, because the correct kind of register is used to store each value, and primitive functions are always called with the correct number and kind of arguments.

Static compilation requires some typing rules in Fig. 3 to explicitly refer to the kinds of types, as described in Fig. 2, that can be assigned to certain expressions. This shows up in the occasional $\tau$ mono-rep and $\tau$ mono-conv side conditions, which we elaborate in Section 4.6. Intuitively, $\tau$ mono-rep captures the fact that $\tau$ has a statically-known, monomorphic representation, and $\tau$ mono-conv says that $\tau$ has a statically-known, monomorphic convention.

\subsection{Simplifying Assumptions}

To maintain a minimal presentation of $\mathcal{I} \mathcal{L}$, we make many simplifying assumptions that reduce its number of features to a small representative core illustrating our main objectives. A realistic implementation will include more features, which can either be added as an extension to $\mathcal{I} \mathcal{L}$, or encoded in terms of the features shown here. Our simplifying assumptions are as follows:

- Higher kinds (e.g., $\star \rightarrow \star)$ are not included, but are a standard extension orthogonal to $\mathcal{I} \mathcal{L}$.

- Int\# is the only exemplary primitive unboxed type, which introduces the only non-pointer representation IntR. Other atomic unboxed types and representations-say, for floating-point numbers, characters, arrays, etc.- can be added straightforwardly, as can primitive operations on these types, like plus\# and minus\# of type Int\# $\leadsto$ Int\# $\leadsto$ Int\#. Unboxed tuple types $\left(\# \tau_{1}, \ldots, \tau_{n} \#\right)$ are an extension that introduces a compound representation roughly dual to the calling convention of functions: $\operatorname{Call}\left[\rho_{1}, \ldots, \rho_{n}\right]$ describes the representations of values that a function needs to consume, while Tuple $\left[\rho_{1}, \ldots, \rho_{n}\right]$ [Eisenberg and Peyton Jones 2017] describes the representations of values that a tuple has within it.

- Int ${ }^{\gamma}$ serves as the only example of an algebraic data type, which happens to be parameterized by a levity $\gamma$ specifying whether the result of the constructor is evaluated eagerly $(U)$ or 
lazily (L). In general, there should be a way to declare new user-defined algebraic data types. These need not be levity polymorphic (i.e., have a $\gamma$ parameter), but $\mathcal{I} \mathcal{L}$ makes it possible to combine levity polymorphism with user-defined data types; see Section 4.8.

- There is no built-in let-binding construct but, as usual, a non-recursive let can be regarded as shorthand for $\lambda$ and application: let $x: \tau=e$ in $e^{\prime} \triangleq\left(\lambda x: \tau . e^{\prime}\right) e$. The typing rules for let-bindings can be derived from the rules for primitive function types $\tau \sim \sigma$. As such, let-bindings inherit similar side conditions on the type of the bound variable; see Section 4.6.

- error is the only source of computational effects in $\mathcal{I L}$ as presented here. Recursive letbindings, which are essential for practical functional programming, can be added straightforwardly, as can other computational effects, such as printing and state as in OCaml, with additional primitive operations.

- Because error is the only side-effect in $\mathcal{I} \mathcal{L}$, call-by-name and call-by-need evaluation always give the same result [Ariola and Felleisen 1997]. So in $\mathcal{I} \mathcal{L}$, we interpret "lifted" (L) as call-byneed for operational concerns, and as call-by-name for equational reasoning. To accommodate richer side-effects, the choice should instead be made explicit. This can be done directly in $I \mathcal{L}$ by further dividing $L$ into separate levities for call-by-name (CBN) and call-by-need (Need) evaluation. For example, evaluating let $x: \operatorname{Int}^{\gamma}=($ print "bye "; I\# 0$)$ in (print "hi "; $\left.x ; x\right)$ prints: "bye hi " with $\gamma=\mathrm{U}$, "hi bye bye " with $\gamma=\mathrm{CBN}$, and "hi bye " with $\gamma=$ Need.

\subsection{Kinds, Representations, Levities, and Conventions}

A kind $\kappa$ has the form TYPE $\rho v$, where $\rho$ describes the representation of the type, and $v$ describes its convention, that is, what operations are allowed on that type. Suppose $x: \tau:$ TYPE $\rho v$; that is, $x$ is a term variable of type $\tau$, whose kind is TYPE $\rho v$. The representation $\rho$ specifies the runtime representation of the value of $x$. Referring to Fig. 1, $\rho$ can be:

- PtrR, meaning that $x$ is a pointer into the garbage-collected heap.

- IntR, meaning that $x$ is a machine integer (not a pointer).

- $r$, a representation variable bound by a $\forall$; that is, we support representation polymorphism [Eisenberg and Peyton Jones 2017].

The convention $v$ describes the allowed operations on $x$, i.e., how it can be consumed. $v$ can be:

- Eval ${ }^{U}$, meaning that $x$ cannot be bound to a computation like $\perp$ (hence $U$ for "Unlifted"). This kind is used for primitive values, and heap pointers that point directly to the value itself (such as a heap-allocated array).

- Eval ${ }^{\mathrm{L}}$, meaning that $x$ may be bound to a computation like $\perp$ (hence $\mathrm{L}$ for "Lifted"). This kind is used for thunks, which might need evaluation to get its value, and might diverge doing so.

- $\mathrm{Eval}^{g}$, where $g$ is a levity variable bound by $\forall$; that is, we support levity polymorphism.

- Call $[\alpha]$, meaning that $x$ is a primitive function (not a thunk) with an arity described by $\alpha$. The arity of an $\mathcal{I} \mathcal{L}$ primitive function might be a fixed non-empty list $\rho_{1}, \ldots, \rho_{m}$, so $x$ takes precisely $m \geq 1$ arguments, ${ }^{7}$ with representations given by $\rho_{i}$. Otherwise, the arity is $\rho_{1}, \ldots, \rho_{m}$, arity $(v)$, meaning that $x$ takes at least $m$ arguments with the listed representations, followed by possibly some more arguments given by the arity of $v$.

- $n$, a convention variable bound by $\forall$; that is, we support convention polymorphism.

The kind TYPE PtrR Eval ${ }^{\mathrm{L}}$ expresses the uniform representation of a value in a lazy language: a pointer to a lifted (i.e., possibly a thunk) object stored in the heap. Because this kind is so common,

\footnotetext{
${ }^{7}$ There is no type in $\mathcal{I} \mathcal{L}$ with the convention Call [], but it can easily arise in extensions of $\mathcal{I} \mathcal{L}$. In practice, unboxed tuple arguments are passed simultaneously in several registers. So the type (\# Bool, Int\#, String \#) $\leadsto$ Int $\mathrm{t}^{\mathrm{L}}$ can be given the kind TYPE PtrR Call[PtrR, IntR, PtrR]. The nullary case of unboxed tuple arguments, $(\# \#) \sim$ Int $^{\mathrm{L}}$, can then be given the kind TYPE PtrR Call[].
} 
we often abbreviate it to $\star$ for the default kind. In a call-by-value language we would instead define the default kind $\star$ as TYPE PtrR Eval ${ }^{\mathrm{U}}$, and $\mathrm{Eval}^{\mathrm{L}}$ would be used sparingly, if at all.

\subsection{Calling Conventions in Kinds}

We track the arity of a function type in its kind, as described in Sections 3.5 and 3.7. For example:

$$
\begin{aligned}
& \text { Int\# } \leadsto \text { Int\# }: \text { TYPE PtrR Call[IntR] } \\
& \text { Int\# } \leadsto \text { Int\# } \leadsto \text { Int\# }: \text { TYPE PtrR Call[IntR, IntR] } \\
& \text { Int\# } \leadsto \text { Int }{ }^{\mathrm{L}} \leadsto \text { Int\# }: \text { TYPE PtrR Call[IntR, PtrR] }
\end{aligned}
$$

The convention of each of these primitive function types has the form $\operatorname{Call}[\alpha]$, where the arity given by $\alpha$ describes the arguments needed to fully call functions of that type.

The formation rule for $\left(\tau_{1} \leadsto \tau_{2}\right)$ keeps track of these arities. Looking at rule Fun in Fig. 2, we see the kind of $\left(\tau_{1} \leadsto \tau_{2}\right)$ has a calling convention of $\operatorname{Call}\left[\rho_{1}\right.$, $\left.\operatorname{arity}\left(v_{2}\right)\right]$. Two special cases are:

$\frac{\Gamma \vdash \tau_{1}: \operatorname{TYPE} \rho_{1} v \quad \Gamma \vdash \tau_{2}: \operatorname{TYPE} \rho^{\prime} \operatorname{Call}\left[\rho_{2}, \ldots, \rho_{m}\right]}{\Gamma \vdash \tau_{1} \leadsto \tau_{2}: \operatorname{TYPE} \operatorname{PtrR} \operatorname{Call}\left[\rho_{1}, \rho_{2}, \ldots, \rho_{m}\right]} \frac{\Gamma \vdash \tau_{1}: \operatorname{TYPE} \rho_{1} v \quad \Gamma \vdash \tau_{2}: \operatorname{TYPE} \rho^{\prime} \operatorname{Eval}^{\gamma}}{\Gamma \vdash \tau_{1} \leadsto \tau_{2}: \operatorname{TYPE} \operatorname{PtrR} \operatorname{Call}\left[\rho_{1}\right]}$

The representation of the first argument $\left(\rho_{1}\right)$ is that of $\tau_{1}$. The rest of the arguments come from the convention $v_{2}$ of $\tau_{2}$, via the type-level operation arity, as defined in Fig. 2. It returns the arity $\alpha$ of $\mathrm{Call}[\alpha]$; and the empty arity in the case of Eval ${ }^{\gamma}$. But $v_{2}$ might also be a variable $n$, and then $\operatorname{arity}(n)$ is stuck; that is why $\operatorname{arity}(v)$ is part of the syntax of $\alpha$ in Fig. 1. Rule K-Conv allows calls to arity to be calculated whenever desired. ${ }^{8}$

\subsection{Polymorphism in Levity and Representation}

We are used to polymorphism over types, but we can gainfully employ polymorphism over levities, representations, and conventions, which is extremely useful in practice. For example, levity polymorphism lets us write some functions that work uniformly over both strict and lazy values. Adding two boxed integers can be defined as

$$
\text { plus : } \left.\left.\forall g_{1} \cdot \forall g_{2} . \forall g_{3} . \text { Int }^{g_{1}} \leadsto \operatorname{Int}^{g_{2}} \leadsto \operatorname{Int}^{g_{3}} \quad \text { plus (I\# } x\right)(\text { I\# } y)=\text { I\# (plus\# } x y\right)
$$

which is short-hand for the following definition in $\mathcal{I L}$ :

$$
\begin{aligned}
& \text { plus : } \forall g_{1} \cdot \forall g_{2} \cdot \forall g_{3} \text {. } \text { Int }^{g_{1}} \leadsto \text { Int }^{g_{2}} \leadsto \text { Int }^{g_{3}} \\
& \text { plus } \left.=\Lambda g_{1} \cdot \Lambda g_{2} \cdot \Lambda g_{3} \cdot \lambda\left(x^{\prime}: \text { Int }^{g_{1}}\right) \cdot \lambda\left(y^{\prime}: \text { Int }^{g_{2}}\right) \text {. case } x^{\prime} \text { of I\# } x \rightarrow \text { case } y^{\prime} \text { of I\# } y \rightarrow \mathrm{I}^{g_{3}} \text { (plus\# } x y\right)
\end{aligned}
$$

Notice that in this definition, the levities $g_{i}$ of the argument and return types are statically unknown, so we must be able to pattern-match on and return values with unknown levities. Specifically, rule INT-E in Fig. 3 allows a case-expression to scrutinize an integer of arbitrary levity $\gamma$. Operationally, a levity-polymorphic case has to test the scrutinee to see if it is a thunk (in case the levity variable is instantiated to L), and if so evaluate it. In essence, we can interpret a case on an unknown levity as a lifted one because a case is always strict and, if it happens that $g$ is $\mathrm{U}$, the branch for handling a thunk is simply dead code. ${ }^{9}$

Similarly, suppose we had a primitive type of arrays, Array\# ${ }^{\gamma}$, with kinding rule

$$
\frac{\Gamma \vdash \gamma \text { lev } \Gamma \vdash \tau: \text { TYPE PtrR } v}{\Gamma \vdash \operatorname{Array} \#^{\gamma} \tau: \text { TYPE PtrR Eval }}
$$

\footnotetext{
${ }^{8}$ Alternatively, we could require $\operatorname{arity}(v)$ to be fully calculated in the Fun kinding rule. This would let us remove $\operatorname{arity}(v)$ from the grammar of arities, but also forces an additional restriction on the formation of types and expressions, specifically FUN and FUN-I, to rule out $\operatorname{arity}(n)$. Such a restriction comes with the cost of breaking a pleasant property of $\mathcal{I} \mathcal{L}$ : except for the $\forall$ quantifier, any type made from well-kinded types is itself well-kinded.

${ }^{9} \mathrm{We}$ assume that the concrete, runtime representation of values (not thunks) is the same for both eager and lazy evaluation This is true in GHC and seems likely in other systems supporting laziness, but it may not hold in some systems.
} 
From a representation point of view, an $A r r a y \# \gamma$ is represented by a pointer and contains pointers. The array itself can be lifted or unlifted, and (independently) can contain lifted or unlifted values. For example, the type Array\# $\#^{\mathrm{L}}\left(\mathrm{Array}^{\mathrm{U}} \mathrm{Int}^{\mathrm{U}}\right.$ ) is a lifted array of pointers, each of which points directly to an array of pointers to (boxed) integers. The ability to exclude the possibility of intermediate thunks in this data structure is very valuable in high-performance code, as a recent spate of GHC proposals shows [Eisenberg 2019; Graf 2020; Martin 2019a,b,c; Theriault 2019].

\subsection{Polymorphism in Convention}

We may also be polymorphic in conventions. Consider the reverse-apply function

$$
\text { revapp } x f=f x
$$

For now, suppose that it returns Int\#. What type should revapp have? Here are two possibilities:

(1) revapp: $\forall\left(t:\right.$ TYPE PtrR Eval $\left.{ }^{\mathrm{L}}\right) . t \leadsto(t \leadsto$ Int\# $) \leadsto$ Int\#

(2) revapp: $\forall\left(t:\right.$ TYPE IntR Eval $\left.{ }^{\mathrm{U}}\right) . t \leadsto(t \leadsto$ Int\# $) \sim$ Int\#

We want to compile a function like revapp to a single block of efficient machine code. To do so, we must know the representation of $x$, because we have to generate instructions to move $x$ around. If $x$ is represented by an integer, it will be passed in one sort of register; if a float, in another; if a pointer then yet another. ${ }^{10}$ So we can choose (1) or (2), but not both.

On the other hand, consider these other possible types:

(3) revapp: $\forall\left(t:\right.$ TYPE PtrR Eval $\left.{ }^{U}\right) . t \leadsto(t \leadsto$ Int\# $) \leadsto$ Int\#

(4) revapp: $\forall(t:$ TYPE PtrR Call[IntR] $) . t \leadsto(t \leadsto$ Int\# $) \leadsto$ Int\#

Since we are simply moving $x$ around, but not otherwise acting upon it, we can simultaneously allow (1), (3), and (4). That is, we can be completely polymorphic in its convention $n$, thus:

(4) revapp: $\forall(n:$ Conv $)(t: \operatorname{TYPE} \operatorname{PtrR} n) . t \leadsto(t \leadsto$ Int\# $) \leadsto$ Int\#

What about the return type of $f$ ? The code for revapp does not manipulate $f$ 's return value at all (it does not even move it around, thanks to tail-call elimination), so it can be completely polymorphic in the representation or $t_{2}$, giving this type:

(5) revapp : $\forall\left(n\right.$ :Conv) $\left(r\right.$ :Rep) ( $g$ :Lev) $\left(t_{1}\right.$ :TYPE PtrR $\left.n\right)\left(t_{2}\right.$ :TYPE $r$ Eval $\left.^{g}\right) . t_{1} \leadsto\left(t_{1} \leadsto t_{2}\right) \leadsto t_{2}$

But notice that, unlike the argument type $t_{1}$, revapp cannot be polymorphic in the convention of $t_{2}$, because a compiler needs to statically know the arity of the $\lambda$-bound argument $f$ to generate code for $(f x)$ inside revapp (Section 4.6). It can, however, be evaluated with any levity.

\subsection{Restrictions on Polymorphism}

Of course we have the usual restrictions on polymorphism, ${ }^{11}$ but $\mathcal{I} \mathcal{L}$ 's polymorphism introduces some new issues. We have already seen how unrestricted polymorphism is incompatible with efficient static code generation ${ }^{12}$ in Section 4.5 , where we cannot allow revapp's type argument $t_{1}$ to have a representation-polymorphic kind. A second restriction is demonstrated by:

$$
\text { twice } f x=f(f x)
$$

Should $(f x)$ be eagerly or lazily evaluated? If it has a lifted type, then we can build a thunk for it, and pass that thunk to $f$. Otherwise, we must evaluate it before the call-remember, variables of

\footnotetext{
${ }^{10}$ Even if pointers occupy the same sort of register as integers, they are treated quite differently by the garbage collector, so the code generator treats them differently.

${ }^{11}$ E.g., every free variable (of every sort) that appears anywhere in the type checking judgment $\Gamma \vdash e: \tau$ must be bound by $\Gamma$.

${ }^{12}$ Runtime code generation would allow the system to clone fresh code for each representationally-distinct instantiation of a function. But this is a pretty big hammer: only .NET does this. To keep things simple, we assume static code generation.
} 
unlifted types are always bound to values, never thunks. So we can give twice either of these types:

(1) twice: $\forall\left(t:\right.$ TYPE PtrR Eval $\left.{ }^{\mathrm{L}}\right) .(t \leadsto t) \leadsto t \leadsto t$

(2) twice: $\forall\left(t:\right.$ TYPE PtrR Eval $\left.{ }^{\cup}\right) .(t \leadsto t) \leadsto t \leadsto t$

but we must choose: unlike revapp, twice cannot be polymorphic in t's convention.

The restrictions on polymorphism used to ensure static compilability are embodied in the shaded premises in the type system of Fig. 3. The judgment $\Gamma \vdash \tau$ mono-rep, defined in Fig. 2 checks that the representation of $\tau$ is monomorphic; that is, that it mentions no variables. This is ensured by the empty context in the second premise of rule MoNo-REP. There is an equivalent judgment $\Gamma \vdash \tau$ mono-conv for conventions. Now returning to Fig. 3 we see the shaded premises:

- Rule Fun-E: for a general application, the argument type must be monomorphic in both the representation (so that we know how to store it while passing), and convention (so that we know when to evaluate it, or for first-class primitive function arguments, how to create it).

- Rule Fun-A-E: in the special case where the argument syntactically has the form of an answer, we can allow the argument type to be levity-polymorphic, since in this case there is no distinction between lazy or eager. If the application is lazy, then the argument does not need to be evaluated anyway. If application is eager, then the argument is already a value (or a variable that must be bound to a value), and again, does not need to be evaluated.

- Rule CLO-I: this rule boxes a primitive function so, just as in Fun-E, we must know that function's arity statically, so we can compile code for it that starts by taking the correct number of arguments.

We can justify these restrictions intuitively, but how do we know that these are the "right" restrictions? To answer that question we will show, in Section 6, how to compile $\mathcal{I} \mathcal{L}$ into our lower level $\mathcal{M L}$. In the translation from $\mathcal{I} \mathcal{L}$ to $\mathcal{M L}$ in Fig. 8, we need exactly the shaded monomorphic restrictions of Fig. 3. If any of these restrictions were removed, then there would be expressions that are well-typed, yet uncompilable.

Our rules also include two additional, unshaded, monomorphism restrictions, in the Fun-I and Fun-A-E rules. These restrictions enforce an extra invariant on the environment $\Gamma$ : every variable in $\Gamma$ has a monomorphic representation. Besides making intuitive sense, this invariant could be necessary in a compiler accounting for more low-level details like storing free variables in a closure; doing so certainly requires knowing their representation. However, perhaps shockingly, the compilation scheme we give in Section 6 does not require any monomorphism restrictions in Fun-I and Fun-A-E: they could be deleted and yet all closed, well-typed expressions could still be compiled. This example suggests different compilers might need different restrictions on polymorphism. And from the reverse standpoint, other compilation schemes might allow for new and more adventurous possibilities for levity, representation, and convention polymorphism.

As an example of why different restrictions are needed for the Fun-E and Fun-A-E rules, recall the encoding of let-bindings from Section 4.1, which gives us the typing rules

$\frac{\Gamma \vdash e: \tau \Gamma, x: \tau \vdash e^{\prime}: \sigma \Gamma \vdash \tau \text { mono-rep } \Gamma \vdash \tau \text { mono-conv }}{\Gamma \vdash \text { let } x: \tau=e \text { in } e^{\prime}: \sigma} \operatorname{LET} \frac{\Gamma \vdash A: \tau \Gamma, x: \tau \vdash e: \sigma \Gamma \vdash \tau \text { mono-rep }}{\Gamma \vdash \text { let } x: \tau=A \text { in } e: \sigma}$ LET-A

derived by composing Fun-I with Fun-E or Fun-A-E, respectively. That is, in general a let can bind a variables to expressions with both a monomorphic representation and convention; but convention-polymorphic answers can also be bound. Neither typing rule is more general than the other: LET-A only applies to some bound expressions (pre-evaluated answers), whereas LET only applies to bindings of some types (ones with statically-known conventions). 
Now, consider these let-bindings, which follow the restrictions of either LET or LET-A above:
(1) let $x: \forall g$. Int $^{g}$ $x=\Lambda g . \mathrm{I}^{g} 0$ in $\ldots$
(3) let $x: \forall n . \forall(t:$ TYPE PtrR $n) . t$ $x=y$ in $\ldots$
(2) let $x:$ Int $^{\mathrm{L}}$ $x=$ plus $\mathrm{L} \mathrm{LL}\left(\mathrm{I}^{\mathrm{L}} 1\right)\left(\mathrm{I} \#^{\mathrm{L}} 2\right)$ in $\ldots$
(4) let $x: \forall g \cdot \operatorname{Int}^{g} \leadsto \operatorname{Int}^{g}$ $x=\Lambda g \cdot \lambda\left(y: \operatorname{Int}^{g}\right) \cdot f\left(f^{\prime} y\right)$ in $\ldots$

Both (1) and (3) bind $x$ to syntactically manifest answers with convention-polymorphic types $\left(\forall g\right.$. Int $^{g}:$ TYPE PtrR Eval ${ }^{g}$ and $\forall n . \forall(t:$ TYPE PtrR $n) . t:$ TYPE PtrR $n$, respectively), which is welltyped by LET-A. In contrast, (2) and (3) bind $x$ to non-answer expressions, so $x$ needs a convention-

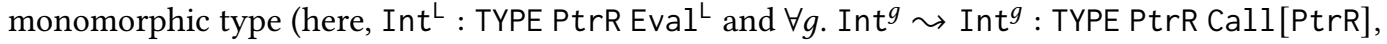
respectively) in order to be well-typed by the LET rule.

\subsection{The Forall Rule}

The polymorphic quantifier $\forall t: \kappa . \sigma$ has no impact on a type's kind: it just inherits the kind of $\sigma$ (rule Forall in Fig. 2). Intuitively, this is because these quantifiers will be totally erased by compilation, and have no impact on the final runtime code; the $\forall$ is "invisible" to the lower-level machine.

However, now that variables may appear in kinds, we must be careful they do not escape their scope. For example, the following type is not well-kinded:

$$
\forall r . \forall\left(t: \text { TYPE } r \text { Eval }{ }^{\mathrm{L}}\right) . t \leadsto t \quad: ? \quad \text { TYPE PtrR Call }[r]
$$

Here the representation $r$ of the first parameter escapes in the calling convention of this primitive function type, because $r$ is meant to be local to the type itself. This nonsense is prevented by the second premise of rule Forall in Fig. 2 and the second premise of rule $\forall$-I in Fig. 3.

\subsection{User-Defined Types and Code Reuse}

As we have seen, polymorphism over these type descriptors-representation, convention, and levityincreases the opportunities for code reuse. We can even have data types that are polymorphic over these descriptors, although this is beyond the scope of the $\mathcal{I} \mathcal{L}$ we formally describe here. For example definition of boxed integers (given in Section 3.2) might be generalized to

$$
\begin{aligned}
& \text { data Int }\left(g: \text { Lev) : TYPE PtrR Eval }{ }^{g}\right. \text { where } \\
& \text { I\# : Int\# } \leadsto \text { Int } g
\end{aligned}
$$

Now, the Int type is parameterized by a chosen levity $(g: \operatorname{Lev})$, which determines whether or not the boxed integers are evaluated eagerly or lazily.

Polymorphism over a constructor's arguments and result can be combined within a single definition. For example, here is a further generalized definition of lists whose spine can be either eagerly or lazily evaluated $(g)$, and containing elements of any arity or evaluation strategy $(n)$ :

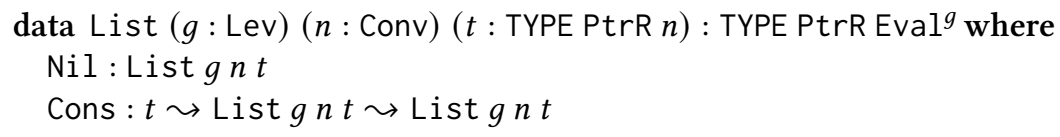

Despite restrictions on polymorphism, we can define some levity-polymorphic functions over this type. For example, we could write the following polymorphic definition which is capable of summing up a list of integers with any combination of levities:

$$
\begin{array}{ll}
\operatorname{sum} & : \forall g_{1} g_{2} g_{3} . \text { List } g_{1} \mathrm{Eval}^{g_{2}}\left(\text { Int } g_{2}\right) \leadsto \text { Int } g_{3} \\
\operatorname{sum} \mathrm{Nil} & =\mathrm{I} \# 0 \\
\operatorname{sum}(\text { Cons }(\mathrm{I} \# x) x s) & =\text { case sum } x s \text { of I\# } y \rightarrow \mathrm{I} \#(\text { plus\# } x y)
\end{array}
$$

sum's caller chooses the levity of the list's spine $\left(g_{1}\right)$, the list's elements $\left(g_{2}\right)$, and the result $\left(g_{3}\right)$. This is possible because sum is completely strict anyway; if it is given an evaluated list or an unevaluated 
Type-based definition of substitutable values: $\Gamma+e$ subst

$\overline{\Gamma \vdash A \text { subst }} \quad \frac{\Gamma \vdash e: \tau \quad \Gamma \vdash \tau: \operatorname{TYPE} \rho \mathrm{Eval}^{\mathrm{L}}}{\Gamma \vdash e \text { subst }} \quad \frac{\Gamma \vdash e: \tau \quad \Gamma \vdash \tau: \operatorname{TYPE} \rho \operatorname{Call}[\alpha]}{\Gamma \vdash e \text { subst }}$

Equational axioms (in each rule, assume that $\Gamma \vdash S$ subst):

\begin{tabular}{|c|c|c|c|}
\hline$(\beta \leadsto)$ & $(\lambda x: \tau . e) S=e[S / x]$ & $(\eta \leadsto)$ & $\lambda x: \tau .(e x)=e: \tau \leadsto \sigma$ \\
\hline$\left(\beta_{\forall}\right)$ & $(\Lambda \chi \cdot e) \phi=e[\phi / \chi]$ & $\left(\eta_{\forall}\right)$ & $\Lambda \chi \cdot(e \chi)=e: \forall \chi \cdot \sigma$ \\
\hline$\left(\beta_{\{\}}\right)$ & $\operatorname{App}\left(\operatorname{Clos}^{\gamma} e\right)=e$ & $\left(\eta_{\{\}}\right)$ & $\operatorname{Clos}^{\gamma}(\operatorname{App} S)=S:{ }^{\gamma}\{\sigma\}$ \\
\hline$\left(\beta_{\text {Int }}\right)$ & $\begin{array}{l}\text { case } \mathrm{I}^{\gamma} A \text { of }=e[A / x] \\
\mathrm{I} \# x \rightarrow e\end{array}$ & $\left(\eta_{\text {Int }}\right)$ & $\begin{array}{l}\text { case } e \text { of } \\
\quad \mathrm{I} \# x \rightarrow \mathrm{I} \#^{\gamma} x\end{array}=e: \mathrm{Int}^{\gamma}$ \\
\hline
\end{tabular}

Plus closure under reflexivity, transitivity, symmetry, and compatibility.

Fig. 4. Equational theory of $\mathcal{I L}$

thunk, the entire thing will be added together before a value is returned. Therefore, the same code can be used for any combination of eager or lazy evaluation.

Other functions, such as the standard definition for mapping over a list:

$$
\operatorname{map} f \mathrm{Nil}=\mathrm{Nil} \quad \operatorname{map} f(\text { Cons } x \times s)=\text { Cons }(f x)(\operatorname{map} f x s)
$$

cannot be so levity polymorphic. That's because the order of evaluation in Cons $(f x)$ (map $f x s)$ depends on the levity of $(f x)$ and the recursive call (map $f x s$ ). We can give this type assignment to map that specifies its result should be lazy, like in Haskell:

map : $\forall g n(a:$ TYPE PtrR $n)\left(b:\right.$ TYPE PtrR Eval $\left.^{\mathrm{L}}\right) .(a \leadsto b) \leadsto$ List $g n a \leadsto$ List L Eval ${ }^{\mathrm{L}} b$

Note that the input list can store values of any convention at all, and like with sum, it can be either spine-strict or spine-lazy; map is strict in its second argument either way. Other evaluation orders for map can be specified by replacing one or both of $L$ in (List $\left.L E v a l^{L} b\right)$ with $U$. Although the definition of map appears the same in $\mathcal{I} \mathcal{L}$, this change will compile to very different machine code.

\subsection{Equational Theory}

The equational theory for $\mathcal{I} \mathcal{L}$, as defined in Fig. 4, gives us a framework to reason about equivalent $I \mathcal{L}$ expressions. We use this as the basis for correctness of compiling a high-level language to $\mathcal{I} \mathcal{L}$ (Theorem 2) and further on to a low-level language (Theorem 3). The rules for function closures (namely $\beta_{\{\}}$and $\left.\eta_{\{\}}\right)$and boxed integers $\left(\beta_{\text {Int }}\right.$ and $\left.\eta_{\text {Int }}\right)$ are unsurprising, as are the rules for erasable abstractions $\left(\beta_{\forall}\right.$ and $\eta_{\forall}$ ). More distinctive is $\eta_{\sim}$, which (as discussed in Section 2) allows unrestricted $\eta$ in either direction for any expression of a primitive function type $\tau \leadsto \sigma$.

That leaves the reduction rule $\beta \leadsto$. As is usual in a call-by-value $\lambda$-calculus, only some expressionscalled substitutable values, denoted by the metavariable $S$-can be passed to a primitive function and substituted for its formal parameter by the $\beta \leadsto$ rule. The $\beta \leadsto$ rule only fires if the argument of the application is substitutable. Unlike most systems-using a purely syntactic definition of substitutable values- $-\mathcal{I} \mathcal{L}$ identifies these expressions by their type and kind, as defined by the rules for the $\Gamma \vdash e$ subst judgment. Using the type and kind of the argument to define substitutability, rather than only its syntax, allows us to integrate several different evaluation orders (call-by-value, call-by-name, etc.) within the same language.

Consider the rules for the $\Gamma \vdash e$ subst judgment. Firstly, we designate all answers $A$ to be substitutable. Notice that, up to type erasure, each answer is considered a value in the call-byvalue $\lambda$-calculus. In other cases, the criteria for substitutability of a typed term $e: \tau$ depends on the convention of $\tau$ rather than the syntax of $e$. For example, in a call-by-name setting, every expression can be substituted for a variable. So in $\mathcal{I} \mathcal{L}$, all lazily-evaluated arguments-which have 
the convention $\mathrm{Eval}^{\mathrm{L}}$-are substitutable values, and hence allow $\beta \leadsto$ to fire. In contrast, the only values in call-by-value languages are answers $(A)$, so variables of type Eval ${ }^{\mathrm{U}}$ can only be substituted with answers. More generally, answers are always substitutable in all of the evaluation strategies we are interested in here, so we can say something more: $A$ is substitutable for all conventions. This extra step is helpful in case we are dealing with an expression-like $x$ or I\#g $i$-which has an unknown convention but will inevitably be substitutable in any case.

For example, consider the different evaluation orders of a function call with lifted or unlifted arguments. On the one hand, we can express a lazy call to plus (Section 4.4) such as

$$
\left(\lambda x: \operatorname{Int}^{\mathrm{L}} . e\right)\left(\text { plus } \mathrm{L}\left(\mathrm{I}^{\mathrm{L}} 1\right)\left(\mathrm{I} \#^{\mathrm{L}} 2\right)\right)=_{\beta_{\sim}} e\left[\text { plus } \mathrm{L}\left(\mathrm{I}^{\mathrm{L}} 1\right)\left(\mathrm{I}^{\mathrm{L}} 2\right) / x\right]
$$

which substitutes the unevaluated argument for the parameter $x$ right away according to $\beta \leadsto$. This is possible because the argument has the type Int ${ }^{\mathrm{L}}$ : TYPE PtrR Eval ${ }^{\mathrm{L}}$, and so it is substitutable by virtue of its type. On the other hand, the corresponding eager call would be

$$
\left(\lambda x: \operatorname{Int}^{\mathrm{U}} . e\right)\left(p l u s \cup\left(\mathrm{I}^{\mathrm{U}} 1\right)\left(\mathrm{I}^{\mathrm{U}} 2\right)\right)=\left(\lambda x: \mathrm{Int}^{\mathrm{U}} . e\right)\left(\mathrm{I}^{\mathrm{U}} 3\right)=\beta_{\sim} e\left[\mathrm{I}^{\mathrm{U}} 3 / x\right]
$$

Here, we cannot apply the $\beta \leadsto$ directly as before, because the argument is not substitutable: it has the type Int ${ }^{\mathrm{U}}$ : TYPE PtrR Eval ${ }^{\mathrm{U}}$ but is not an answer. Instead we must first evaluate the argument; the result is the answer $\left(\mathrm{I}^{\cup} 3\right)$, and $\beta \leadsto$ can now fire.

\section{COMPILATION TO $\mathcal{I} \mathcal{L}$ FROM A HIGHER LEVEL}

$I \mathcal{L}$ is, by design, a fairly low-level language making fine distinctions about representation, calling conventions, evaluation orders, and so on. This makes it a target for both eager and lazy languages. To see how, we now give translations for call-by-name and call-by-value System $\mathrm{F}$ into $\mathcal{I} \mathcal{L}$.

\subsection{Call-by-Name System F to $\mathcal{I L}$}

To translate call-by-name System $\mathrm{F}$ into $\mathcal{I} \mathcal{L}$, we begin by picking a single "uniform" $\mathcal{I} \mathcal{L}$ kind $\star$ that captures all the types of the source language, namely $\star=$ TYPE PtrR Eval ${ }^{\mathrm{L}}$. Each source-language type $\tau$ translates to an $\mathcal{I} \mathcal{L}$ type $\llbracket \tau \rrbracket$ of kind $\star$; that is, a pointer to a lifted value, perhaps a thunk.

Fig. 5 gives this type translation. To get the correct call-by-name semantics for numbers, we use the boxed integer type Int ${ }^{\mathrm{L}}$, which happily has the correct kind. However, even though the primitive function type $\llbracket \tau \rrbracket \sim \llbracket \sigma \rrbracket$ has the correct call-by-name semantics, it has the wrong kind TYPE PtrR Call[PtrR], so the translation coerces Call[PtrR] to Eval ${ }^{\mathrm{L}}$ with a closure type. Polymorphic type abstraction is unchanged, only clarified that bound type variable ranges over $\star$.

To compile expressions, we only need to expand out the additions prescribed by the translation of types. Numeric constants need to be boxed, functions and their calls need the explicit coercions to and from closures, and bound type variables are annotated with their uniform kind.

\subsection{Call-by-Value System F to $\mathcal{I} \mathcal{L}$}

We can compile a call-by-value version of system $\mathrm{F}$ using virtually the same procedure as above. Again, we need to decide on a uniform kind that is suitable for each source-level type, which is $\star=$ TYPE PtrR Eval U for call-by-value evaluation. As before, we can compile source-level types following the invariant that $\llbracket \tau \rrbracket: \star$, as again shown in Fig. 5.

Note that we still compile integers to a boxed type, so that all values are represented uniformly by a pointer, but this time we make it unlifted to reflect the call-by-value semantics. Function types are still wrapped in a closure, as in the call-by-name case, but this time unlifted ones.

The translation of polymorphism is more complex, however, due a mismatch with the semantics of call-by-value System F. With call-by-value evaluation, the abstraction $\Lambda t . \perp$ is a value, even though $\perp$ diverges, whereas in call-by-name they would be $\eta$-equivalent. We need to make sure 
Call-by-name where $\star=$ TYPE PtrR Eval ${ }^{\mathrm{L}}$

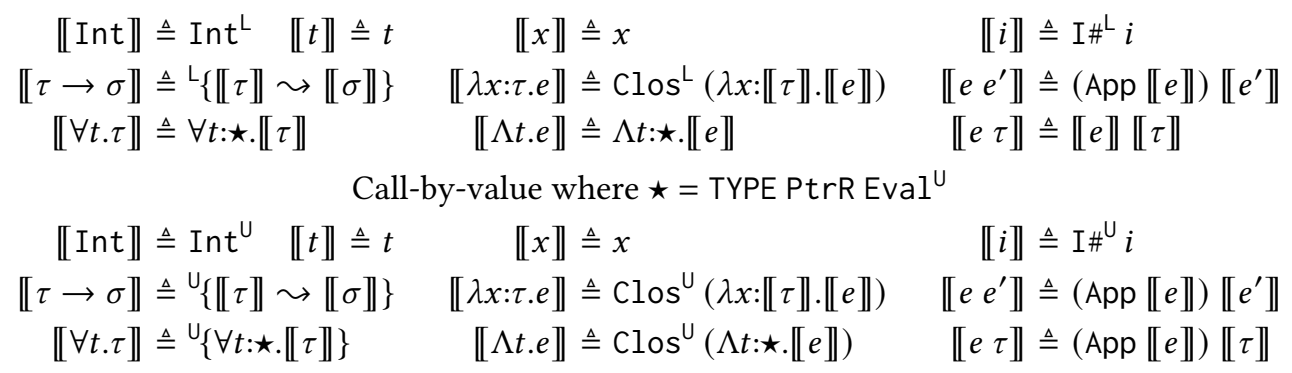

Fig. 5. Compiling call-by-name and call-by-value System F to $\mathcal{I L}$

Call-by-name substitutable values:

$$
V::=e
$$

Equational axioms (for both call-by-name and -value definitions of $V$ ):
Call-by-value substitutable values:

$$
V::=x|\lambda x: \tau . e| \Lambda t . e
$$

$\left(\beta_{\rightarrow}\right)$

$\left(\beta_{\forall}\right)$

$$
\begin{aligned}
(\lambda x: \tau . e) V & =e[V / x] \\
(\Lambda t . e) \tau & =e[\tau / t]
\end{aligned}
$$

(name)

Fig. 6. Equational theory of System F; call-by-value and call-by-name

that this abstraction is still a value even after the polymorphic $\Lambda$ is erased. For that reason, we must introduce the additional call-by-value closure which is preserved to runtime.

The compilation of call-by-value expressions is nearly the same as call-by-name expressions. Besides swapping $L$ for $U$, the only difference is in for polymorphic abstractions and instantiations. These have an extra closure that signifies call-by-value evaluation and, more importantly, makes sure that the value $\Lambda t . e$ in System F compiles to a value, namely $\mathrm{Clos}^{\bigcup}(\Lambda t: \star$. $\llbracket e \rrbracket)$, which is essential when side effects or non-termination enters the picture. For example, evaluating $\Lambda t$.error $t i$ returns immediately in call-by-value System $\mathrm{F}$, but the corresponding $\Lambda t$ : $\star$.error $\mathrm{PtrR} \cup t i$ causes an error in $\mathcal{I} \mathcal{L}$. Intuitively, the explicit closure and application serve to codify the standard definition of type erasure for call-by-value System F, which traditionally erases $\Lambda$ t.e into $\lambda($ ).e.

\subsection{Correctness of Source-to- $\mathcal{I} \mathcal{L}$ Compilation}

Compiling call-by-name and call-by-value System $\mathrm{F}$ into $\mathcal{I} \mathcal{L}$ are both correct: type checking and equalities are preserved by translation. We assume the standard type system for System $\mathrm{F}$ and semantics in Fig. 6. Note that the name axiom, which gives a name to the argument of a function, corresponds to a right-to-left evaluation order for the call-by-value semantics, and is a consequence of $\beta \rightarrow$ in the call-by-name semantics. The preservation of types is straightforward, where the compilation of a typing environment $\llbracket \Gamma \rrbracket$ is defined pointwise.

Theorem 1 (Type Preservation). If $\Gamma \vdash e: \tau$ is derivable then so is $\llbracket \Gamma \rrbracket \vdash \llbracket e \rrbracket: \llbracket \tau \rrbracket$.

Theorem 2 (Soundness and Completeness). $\Gamma \vdash e=e^{\prime}: \tau$ in call-by-name System $F$ if and only if $\llbracket \Gamma \rrbracket \vdash \llbracket e \rrbracket=\llbracket e^{\prime} \rrbracket: \llbracket \tau \rrbracket$ via call-by-name compilation, and likewise for call-by-value.

\section{COMPILATION FROM $\mathcal{I L}$ TO A LOWER LEVEL}

In order to illustrate how our levity-polymorphic intermediate language $\mathcal{I} \mathcal{L}$ might be compiled to a more conventional, lower-level language, we introduce an abstract machine that supports 


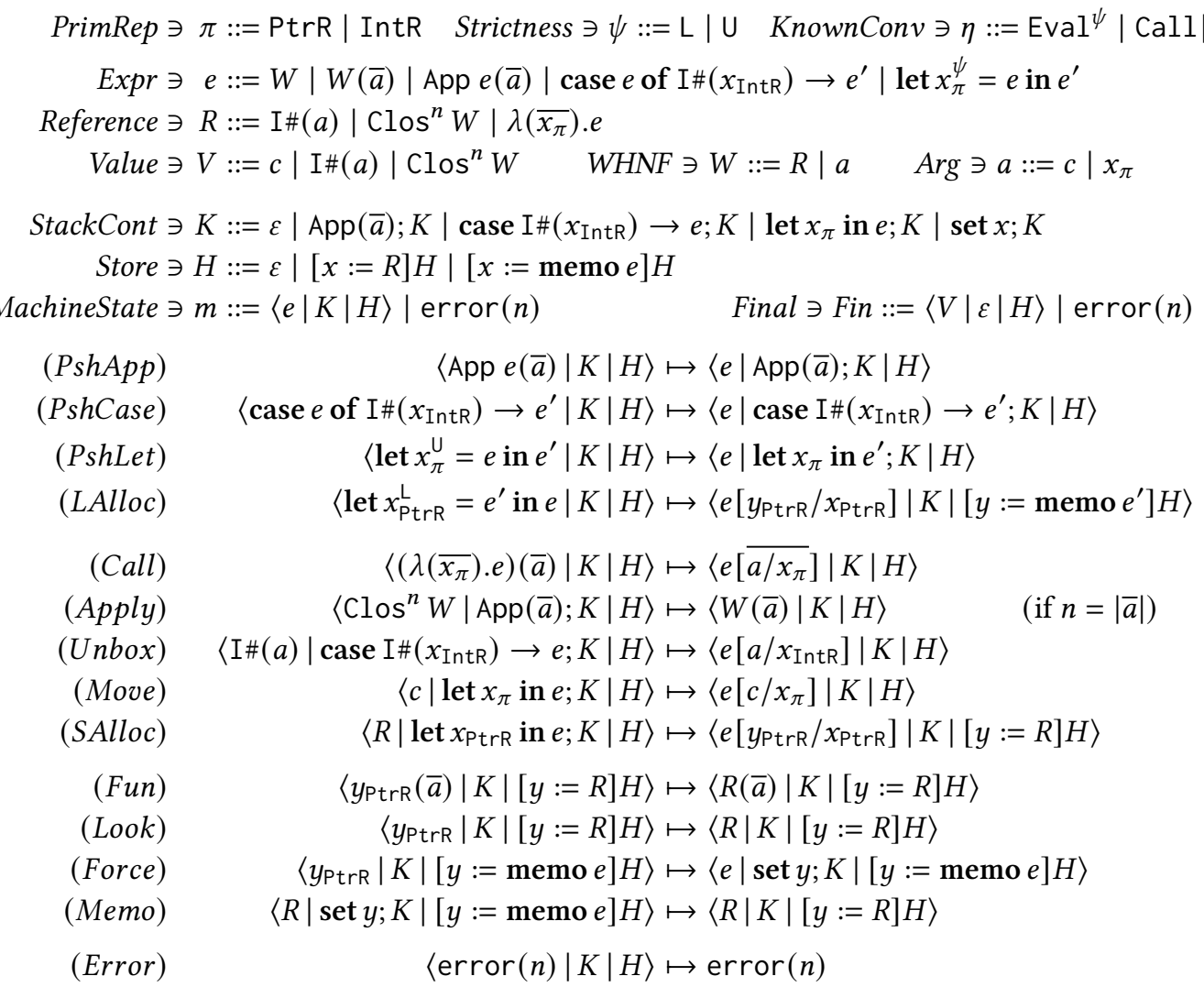

Fig. 7. Syntax and semantics for $\mathcal{M L}$

non-uniform representations and function arities, both of which must be monomorphic. The idea is to model a realistic machine architecture with multiple basic kinds of data representations (e.g., pointers vs. integers), and where primitive functions are passed multiple arguments but are otherwise first order (though primitive function pointers may be passed as arguments and invoked). The syntax of this language, which we call $\mathcal{M} \mathcal{L}$, is given in Fig. 7, along with its abstract machine. ${ }^{13}$

The syntax of $\mathcal{M L}$ is restricted from the more $\lambda$-calculus-inspired $\mathcal{I} \mathcal{L}$ in several ways:

- Expressions follow the A-normal form (ANF) convention [Sabry and Felleisen 1993]: all arguments $a$ are either variables or constants. To support ANF, $\mathcal{M L}$ has a let construct.

- Primitive functions $\left(\lambda\left(\overline{y_{\pi}}\right) . e\right)$ and calls $(W(\bar{a}))$ have an explicit arity and pass multiple arguments at once, but cannot be partially applied.

- An applied function cannot be an arbitrary expression; it must be a weak head-normal form, namely a reference to a $\lambda$, variable, or constant. Every application $W(\bar{a})$ can be resolved in at most two steps: lookup $W$ if it's a variable, then apply $W$ if it's a $\lambda$ or constant (like error).

As such, it is impossible to chain several calls in a row. For example, $f(1)(2)$ is not a legal expression in $\mathcal{M L}$. If $f$ is an arity 2 primitive function, it must be called as $f(1,2)$; if it is an arity-1 primitive function returning a closure of an arity-1 primitive function, it must be called as $(\operatorname{App} f(1))(2)$.

\footnotetext{
${ }^{13}$ For aficionados of GHC, $\mathcal{I} \mathcal{L}$ is like GHC's Core language, while $\mathcal{M L}$ is like the STG language [Peyton Jones 1992].
} 
The number of arguments passed at once is explicitly fixed in each $\lambda$-abstraction and call site. In other words, $\mathcal{M} \mathcal{L}$ does not support polymorphism of function arity.

$\mathcal{M} \mathcal{L}$ 's syntax includes annotations that make the semantically important information in types explicit, so the syntax of programs makes it clear how they are executed. In particular, each variable is annotated with its representation $\pi$, which must be either a pointer (PtrR) or integer (IntR), corresponding to an assignment to an appropriate register. In other words, all variables are permanently assigned a representation-intuitively, stored in either an integer or address register. By design, $\mathcal{M L}$ does not support polymorphism over these representations because the different types of machine registers are distinct, and the choice made is fixed in the code.

Additionally, each let binding is annotated as either eager $(U)$ or lazy $(L)$. This controls whether the right-hand side of the let is evaluated first before being bound to the variable, or bound first and evaluated later as needed. Again, this decision about evaluation order must be statically chosen for each let, so $\mathcal{M L}$ does not support polymorphism over evaluation order.

\subsection{The Semantics of $\mathcal{M L}$}

Executing an $\mathcal{M} \mathcal{L}$ program involves a machine configuration of the form $\langle e|K| H\rangle$, where $e$ is the expression being evaluated, $K$ is the continuation or call stack of evaluation, and $H$ is the heap for storing allocated memory. Heaps may contain both values $([x:=V] H)$ or unevaluated thunks $([x:=\operatorname{memo} e] H)$. Both call stacks and heaps are conventional, except that a stack may contain an application of many arguments in a single stack frame, like $\operatorname{App}(\bar{a}) ; K$. The other cases of stack frames include a case, a strict let binding, and a set construct to memoize thunk evaluation.

Many of the steps of the machine are also conventional, including those for pushing stack frames (PshApp, PshCase, PshLet) and allocating memory (LAlloc, SAlloc), but note that we include cases for both lazy bindings (LAlloc) and strict ones (PshLet, SAlloc). Next we have the rules for performing interesting reductions. Apply resolves the application of a closure by extracting the primitive function it contains, and Call calls a primitive function directly. Note that Apply can check that the number of arguments matches the arity of the closure at runtime (and potentially respond appropriately if they do not match, as we do later in Section 7). Instead, Call is merely undefined when the arguments don't match the bound parameters, representing a type or memory unsafe error. In addition, we have Move for moving a constant into an appropriate variable (corresponding to a register) and Unbox for extracting the contents of a boxed integer. Finally, we have the rules for handling pointer variables at runtime. Fun expects a function pointer to map to a value. For other pointers, we have to check if it is evaluated, to either Look up values or else Force thunks. ${ }^{14}$ When a forced thunk returns a value, it is Memoized to share the result on future uses.

\subsection{Compilation}

Compilation from $\mathcal{I} \mathcal{L}$ to the low-level machine language $\mathcal{M L}$ is given in Fig. 8. The top-level translation is $\mathcal{E}_{v} \llbracket e \rrbracket_{\theta}^{\Gamma}$, which compiles a typed expression $\Gamma \vdash e: \tau$ given $\tau$ 's convention is $v$. The environment $\theta$ is a mapping from $\mathcal{I} \mathcal{L}$ variables to $\mathcal{M L}$ arguments (either constants or representation-annotated variables) written as $\left[a_{1} / x_{1}\right] \ldots\left[a_{n} / x_{n}\right]$.

A key part in understanding the compilation in Fig. 8 is to remember the distinction between calling and evaluating. In our system, only expressions with types like Int\#, Int ${ }^{\gamma}$, and ${ }^{\gamma}\{\tau\}$ can be evaluated. In contrast, expressions with types like $\tau \leadsto \sigma$ can only be called. Implementing this distinction is the main role of $\mathcal{E}_{v} \llbracket e \rrbracket_{\theta}^{\Gamma}$, which takes into account the convention $v$ of $e$ : if it is Eval ${ }^{\gamma}$

\footnotetext{
${ }^{14}$ Note that this uniform check on pointers $y_{\mathrm{P} t r R}$ is needed to support levity polymorphism for types like $\operatorname{Int}^{g}$ and ${ }^{g}\{\tau\}$. In a more practical compiler, we could have specialized code that avoids a check when it is statically known, due to type checking that $y_{\mathrm{P} t r R}$ must be unlifted, so that the Look step always applies without a dynamic check. Thus, a language which is call-by-value by default does not have to pay the runtime penalty for thunks unless they are actually being used.
} 


\section{In the following, all equations are tried left-to-right, top-to-bottom.}

Top-level eta expansion:

$$
\begin{aligned}
& \mathcal{E}_{v} \llbracket A \rrbracket_{\theta}^{\Gamma} \triangleq \mathcal{A} \llbracket A \rrbracket_{\theta}^{\Gamma} \quad \mathcal{E}_{\mathrm{Eval}^{\gamma}} \llbracket e \rrbracket_{\theta}^{\Gamma} \triangleq C \llbracket e \rrbracket_{\theta}^{\Gamma}(\varepsilon) \quad \mathcal{E}_{\mathrm{Call}[\bar{\pi}]} \llbracket e \rrbracket_{\theta}^{\Gamma} \triangleq \lambda\left(\overline{x_{\pi}}\right) \cdot C \llbracket e \rrbracket_{\theta}^{\Gamma}\left(\overline{x_{\pi}}\right) \\
& \text { Constants and variables: } \\
& \left.C \llbracket i \rrbracket_{\theta}^{\Gamma}(\varepsilon) \triangleq i \quad C \llbracket x \rrbracket_{\theta}^{\Gamma, x: \tau}(\varepsilon) \triangleq \theta(x) \quad \text { (if } \Gamma \vdash \tau \stackrel{\text { conv }}{\rightsquigarrow} \mathrm{Eval}^{\psi}\right) \\
& C \llbracket \operatorname{error} \rrbracket_{\theta}^{\Gamma}(a) \triangleq \operatorname{error}(a) \quad C \llbracket x \rrbracket_{\theta}^{\Gamma, x: \tau}(\bar{a}) \triangleq(\theta(x))(\bar{a}) \quad(\text { if } \Gamma \vdash \tau \stackrel{\text { conv }}{\rightsquigarrow} \operatorname{Call}[\bar{\pi}]) \\
& C \llbracket \operatorname{App} e \rrbracket_{\theta}^{\Gamma}(\bar{a}) \triangleq \operatorname{App}\left(C \llbracket e \rrbracket_{\theta}^{\Gamma}(\varepsilon)\right)(\bar{a}) \\
& C \llbracket e \phi \rrbracket_{\theta}^{\Gamma}(\bar{a}) \triangleq C \llbracket e \rrbracket_{\theta}^{\Gamma}(\bar{a}) \\
& C \llbracket e A \rrbracket_{\theta}^{\Gamma}(\bar{a}) \triangleq C \llbracket e \rrbracket_{\theta}^{\Gamma}\left(\mathcal{A} \llbracket A \rrbracket_{\theta}^{\Gamma}, \bar{a}\right) \\
& C \llbracket e A \rrbracket_{\theta}^{\Gamma}(\bar{a}) \triangleq \text { let } x_{\mathrm{PtrR}}^{U}=\mathcal{A} \llbracket A \rrbracket_{\theta}^{\Gamma} \text { in } C \llbracket e \rrbracket_{\theta}^{\Gamma}\left(x_{\mathrm{PtrR}}, \bar{a}\right) \\
& C \llbracket e e^{\prime} \rrbracket_{\theta}^{\Gamma}(\bar{a}) \triangleq \operatorname{let} x \frac{\operatorname{lev}(\eta)}{\pi}=\mathcal{E}_{\eta} \llbracket e^{\prime} \rrbracket_{\theta}^{\Gamma} \text { in } C \llbracket e \rrbracket_{\theta}^{\Gamma}\left(x_{\pi}, \bar{a}\right) \quad\left(\text { if } \Gamma \vdash e^{\prime}: \tau, \Gamma \vdash \tau \stackrel{\text { rep }}{\sim} \pi \text {, and } \Gamma \vdash \tau \stackrel{\text { conv }}{\rightsquigarrow} \eta\right) \\
& \text { Boxing and unboxing: } \\
& C \llbracket \mathrm{I} \#^{\gamma} A \rrbracket_{\theta}^{\Gamma}(\varepsilon) \triangleq \mathcal{A} \llbracket \mathrm{I} \#^{\gamma} A \rrbracket_{\theta}^{\Gamma} \\
& C \llbracket \mathrm{I} \#^{\gamma} e \rrbracket_{\theta}^{\Gamma}(\varepsilon) \triangleq \text { let } x_{\mathrm{IntR}}^{\mathrm{U}}=C \llbracket e \rrbracket_{\theta}^{\Gamma}(\varepsilon) \text { in } \mathrm{I} \#\left(x_{\mathrm{IntR}}\right) \\
& C \llbracket \text { case } e^{\prime} \text { of I\# } x \rightarrow e \rrbracket_{\theta}^{\Gamma}(\bar{a}) \triangleq \operatorname{case} C \llbracket e^{\prime} \rrbracket_{\theta}^{\Gamma}(\varepsilon) \text { of I\# }\left(x_{\operatorname{IntR}}\right) \rightarrow C \llbracket e \rrbracket_{\left[x_{\operatorname{IntR}} / x\right] \theta}^{\Gamma, x: \operatorname{Int} \#}(\bar{a}) \\
& \text { Abstractions: } \\
& C \llbracket \lambda x: \sigma . e \rrbracket_{\theta}^{\Gamma}\left(a^{\prime}, \bar{a}\right) \triangleq C \llbracket e \rrbracket_{\left[a^{\prime} / x\right] \theta}^{\Gamma, x \cdot \sigma}(\bar{a}) \\
& C \llbracket \Lambda \chi \cdot e \rrbracket_{\theta}^{\Gamma}(\bar{a}) \triangleq C \llbracket e \rrbracket_{\theta}^{\Gamma, \chi}(\bar{a}) \\
& C \llbracket \operatorname{Clos}^{\gamma} e \rrbracket_{\theta}^{\Gamma}(\varepsilon) \triangleq \mathcal{A} \llbracket \operatorname{Clos}^{\gamma} e \rrbracket_{\theta}^{\Gamma} \\
& \mathcal{A} \llbracket c \rrbracket_{\theta}^{\Gamma} \triangleq c \quad \mathcal{A} \llbracket x \rrbracket_{\theta}^{\Gamma} \triangleq \theta(x) \quad \mathcal{A} \llbracket \mathrm{I} \#^{\gamma} A \rrbracket_{\theta}^{\Gamma} \triangleq \mathrm{I} \#\left(\mathcal{A} \llbracket A \rrbracket_{\theta}^{\Gamma}\right) \\
& \mathcal{A} \llbracket A \phi \rrbracket_{\theta}^{\Gamma} \triangleq \mathcal{A} \llbracket A \rrbracket_{\theta}^{\Gamma} \quad \mathcal{A} \llbracket \Lambda \chi \cdot A \rrbracket_{\theta}^{\Gamma} \triangleq \mathcal{A} \llbracket A \rrbracket_{\theta}^{\Gamma} \\
& \mathcal{A} \llbracket \operatorname{Clos}^{\gamma} e \rrbracket_{\theta}^{\Gamma} \triangleq \mathrm{Clos}|\operatorname{arity}(\eta)| \mathcal{E}_{\text {Call } \mid \operatorname{arity}(\eta)]} \llbracket e \|_{\theta}^{\Gamma} \quad \text { (if } \Gamma \vdash e: \tau \text { and } \Gamma \vdash \tau \stackrel{\text { conv }}{\rightsquigarrow} \eta \text { ) }
\end{aligned}
$$

Calculating known representations and conventions, and the levity of a known convention:

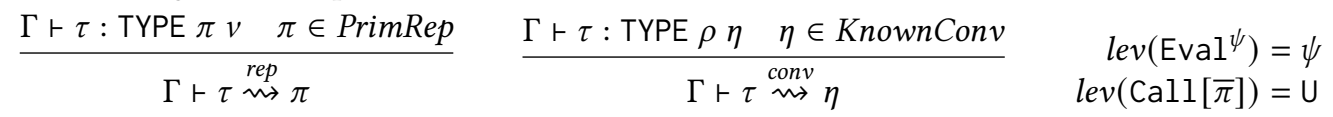

Fig. 8. Compiling $\mathcal{I} \mathcal{L}$ to $\mathcal{M L}$

then we can evaluate the result of $e$ directly by the main compilation translation, otherwise if it is $\operatorname{Call}[\bar{\pi}]$ then $e$ must be called (not evaluated). To make sure that the definition and call sites of a primitive function match, we always fully $\eta$-expand these expressions when they are defined: either on the right-hand side of lets or in the body of Closures. Because of $\eta$-expansion, this step of compilation is only defined when the calling convention is statically known (e.g., it is not a variable $n$ or partially-defined like Call $\left[r_{1}, r_{2}\right.$, arity $\left.\left.(n)\right]\right)$. In any case, we next move to the main work-horse of compilation, $C \llbracket e \rrbracket_{\theta}^{\Gamma}(\bar{a})$, that produces $\mathcal{M L}$ code to evaluate the result of $e$ applied to the arguments $(\bar{a})$. Again, there are invariants to this translation that we will enumerate shortly.

For $C \llbracket e \rrbracket_{\theta}^{\Gamma}(\bar{a})$, literal constants are just passed through, but compiling a call to error assumes precisely one argument. What if the user has written a partial application of error? Such partial 
applications are always $\eta$-expanded to be fully saturated, satisfying the requirement here. Compiling a variable $x$ looks it up in the environment $\theta$, but there is different $\mathcal{M L}$ code for evaluating $x$ versus calling it, even when there are no arguments. In other words, a primitive function variable $x$ with the empty calling convention Call[] compiles as $C \llbracket x \rrbracket_{\left[y_{\mathrm{PtrR}} / x\right] \theta}^{\Gamma(\varepsilon)=y_{\mathrm{PtrR}}()-\text { a nullary }}$ function call-but a variable $x$ with the convention $\mathrm{Eval}^{\gamma}$ compiles as to the pointer lookup

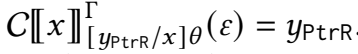

Closure applications are compiled straightforwardly, and erasable arguments $\phi$ and binders $\Lambda \chi . e$ are simply dropped. $\mathcal{I} \mathcal{L}$ answers can be compiled outright to a $\mathcal{M L}$ WHNF via $\mathcal{A} \llbracket A \rrbracket_{\theta}^{\Gamma}$. Compiling an application to an answer $A$ depends on the nature of that answer:

- If $\mathcal{A} \llbracket A \rrbracket_{\theta}^{\Gamma}$ is a variable or constant (after type erasure), then it can be passed directly.

- Otherwise, name the argument with a let (respecting the A-normal form) and pass it by reference. In this case, the compiled argument will always have the form I\#(a) or $\operatorname{Clos}^{n} W$, meaning the let-binding will always be represented as a pointer into the heap. ${ }^{15}$

In the variable case, we do not need to track the levity or representation of the argument, because these decisions have already been made by the context, when the variable definition itself was compiled. Crucially, we did not have to look up any information in the typing environment to compile answer arguments; this is why no highlighted premises are needed in rule Fun-A-E.

In the case of an application $e e^{\prime}$ to an arbitrary argument that needs to be computed, corresponding to Fun-E, we always generate a let similar to the second case for $e$ A. However, for Fun-E, we need to determine the representation, convention, and levity of the binding, which could truly be anything. This corresponds to the highlighted side conditions in Fun-E.

\subsection{Correctness of Compilation}

Notice how the same polymorphism restrictions used in the typing rules also appear during compilation. Even though the defined compilation translation is partial (not every syntactically valid expression can be compiled), all well-typed $\mathcal{I} \mathcal{L}$ expressions with a known convention have a defined compilation to $\mathcal{M L}$. In particular, $\mathcal{E}_{\eta} \llbracket e \rrbracket$ is well-defined for any closed expression $\vdash e: \tau:$ TYPE $\rho \eta$, where the syntax of known conventions $\eta$ is given in Fig. 7 .

In fact, we allow for a little more levity polymorphism during compilation: $\mathcal{E}_{\mathrm{Eval}}$ 【e $e$, for a polymorphic levity $g$, is also allowed. That's because the generated code will be executed exactly when expression is evaluated: in other words, when a computation is forced, there is no difference between eager $(U)$ or lazy $(L)$. This added flexibility is essential for compiling levity polymorphic expressions appearing in strict contexts, such as in the discriminant of a case or first argument of App. Although implicit, the $C$ translation assumes that evaluation of the compiled expression is being forced. In contrast, the $\mathcal{A}$ translation does not assume this, because it is used in contexts that do not force the expression. This small difference is how we are able to pass variables of any convention (eager, lazy, or primitive functions) without erroneously introducing extra strictness.

During compilation, we occasionally need to know representation $(\pi)$ or convention $(\eta)$ of a sub-expression. This appears in Fig. 8 as highlighted side conditions $\Gamma \vdash \tau \stackrel{\text { rep }}{\rightsquigarrow} \pi$ and $\Gamma \vdash \tau \stackrel{\text { conv }}{\rightsquigarrow} \eta$, respectively. In general, compilation could fail if the representation or convention in the kind of $\tau$ are partially unknown-that is, contains free variables. But any closed representation has the form $\pi$ and any closed convention is equivalent to a $\eta$, as defined by the syntax of $\mathcal{M L}$ in Fig. 7 . The places where this requirement appears corresponds exactly to the highlighted monomorphism restrictions in Fig. 3.

\footnotetext{
${ }^{15} \mathrm{~A}$ more feature-rich language may allow for representations other than just a single pointer, in this case. Even then, answers compile to values with syntactically manifest representations, so no additional typing information is needed here.
} 
Theorem 3 (Closed Compilation). If $\vdash e: \tau$ and $\vdash \tau:$ TYPE $\rho v$ then $\mathcal{E}_{v} \llbracket e \rrbracket$ is defined.

This theorem just states when compilation succeeds in generating $\mathcal{M L}$ code from a closed $\mathcal{I} \mathcal{L}$ expression. We should also expect that compilation preserves the behavior of that $\mathcal{I} \mathcal{L}$ expression as well. In other words, if an expression is equal to some answer in $\mathcal{I} \mathcal{L}$ (as per Fig. 4), then executing the compiled code should give the same answer in $\mathcal{M L}$. But we are not interested in evaluating primitive functions directly-they are called, not evaluated!-so answers will be of some Evaluatable types like (un)boxed integers, which are simple enough values to line up on the nose.

Theorem 4 (Soundness and Completeness).

(1) For any $\vdash e$ : Int\#, $\vdash e=i$ : Int\# if and only if $\left\langle\mathcal{E}_{\mathrm{Eval}^{\mathrm{U}}} \llbracket e \rrbracket|\varepsilon| \varepsilon\right\rangle \mapsto^{*}\langle i|\varepsilon| H\rangle$.

(2) For any $\vdash e: \operatorname{Int}^{\gamma}, \vdash e=\mathrm{I}^{\gamma} i:$ Int $^{\gamma}$ if and only if $\left\langle\mathcal{E}_{\mathrm{Eval}^{\gamma}} \llbracket e \rrbracket|\varepsilon| \varepsilon\right\rangle \mapsto^{*}\langle\mathrm{I} \#(i)|\varepsilon| H\rangle$.

\section{DYNAMIC ARITY}

Consider the following program written in Haskell, where exp is some expensive function: ${ }^{16}$

$$
\begin{aligned}
& \text { data } T=M k T(\text { Int } \rightarrow \text { Int } \rightarrow \text { Int }) \\
& t 1=\operatorname{MkT}(\lambda x \text {. let } z=\exp x \text { in } \lambda y . z+y) \\
& t 2=M k T \text { plus }
\end{aligned}
$$

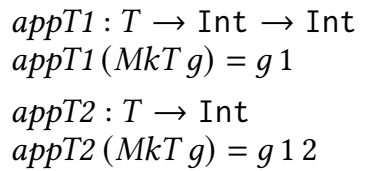

In terms of the informal notion of arity in the source language (Section 2), we can say that $t 1$ stores an arity 1 closure and $t 2$ stores an arity 2 closure. Likewise, appT1 performs an arity 1 application and appT2 performs an arity 2 application. This can be compiled to $\mathcal{I} \mathcal{L}$ (extended with data type declarations) similar to the translation in Fig. 5, which formalizes the arities like so:

$$
\begin{aligned}
& \left.\operatorname{data} T=M k T^{\mathrm{L}_{\{}} \text {Int } \leadsto{ }^{\mathrm{L}}\{\text { Int } \leadsto \text { Int }\}\right\} \\
& t 1=M k T\left(\operatorname{Clos}^{\llcorner} \lambda x \text {. let } z=\exp x \text { in } \operatorname{Clos}^{\llcorner} \lambda y . z+y\right) \\
& t 2=\operatorname{MkT}\left(\operatorname{Clos}^{\mathrm{L}} \lambda x \cdot \mathrm{Clos}^{\mathrm{L}} \lambda y \cdot p l u s x y\right) \\
& \begin{array}{l}
\text { appT1:T } \sim \mathrm{L}_{\{\text {Int } \sim \text { Int }\}} \\
\operatorname{appT1}(M k T g)=\operatorname{App} g 1 \\
\text { appT2 }: T \leadsto \text { Int } \\
\text { appT2 }(M k T g)=\operatorname{App}(\operatorname{App} g 1) 2
\end{array}
\end{aligned}
$$

Notice how appT2 ( $M k T g)$ does not apply $g$ to both arguments at once; instead, it must evaluate $g$ applied to 1 first, then apply the returned closure to 2 in a separate step. The two-step application process is mandated by the type of the $M k T$ constructor, even though most of the time $M k T$ will ultimately be used store a closure capable of accepting both arguments at once. This arity demotion can be seen in $t 1$, where the binary plus function is wrapped up in a chain of two unary closures, as required by $M k T$. As a result, the call appT1 $t 2$ must pass 1 and 2 separately to plus, losing the opportunity for the faster binary calling convention that seemed possible in the source program.

We can attempt improve the performance of $t 2$ and $a p p T 2$ with an alternate translation:

$$
\begin{aligned}
& \text { data } \left.T^{\prime}=M k T^{\prime} \mathrm{L}_{\{\text {Int }} \leadsto \text { Int } \leadsto \text { Int }\right\} \\
& t 1^{\prime}=M k T^{\prime}\left(\operatorname{Clos}^{L} \lambda x . \lambda y \text {. let } z=\exp x \text { in } z+y\right) \\
& t 2^{\prime}=M k T^{\prime}\left(\operatorname{Clos}^{\mathrm{L}} \text { plus }\right)
\end{aligned}
$$

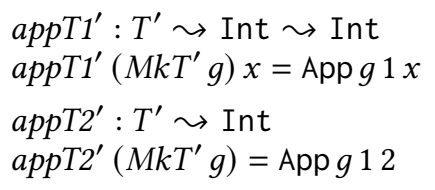

Now, $M k T^{\prime}$ contains a closure of a single binary function. This way, appT2' $t 2^{\prime}$ steps to the single binary application plus 12 , passing both arguments at once to plus. But the type of $M k T^{\prime}$ makes it impossible to contain closures that memoize work when applied to only one argument-an unfortunate, unintended ramification of this "optimization." Unlike before, the closure inside $t 1^{\prime}$ recomputes $\exp x$ every time the second argument is given, instead of memoizing the result once the first argument is provided. Here, we make this fact syntactically explicit by $\eta$-expanding the

\footnotetext{
${ }^{16}$ The exact same example applies to OCaml as well by replacing $\mathrm{L}$ with $\mathrm{U}$ in translations into $\mathcal{I} \mathcal{L}$ that follow.
} 
definitions of $t 1^{\prime}$ and $a p p T 1^{\prime}$, but because $\leadsto$-types are fully extensional, the same recomputation

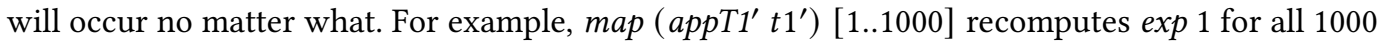
elements of the list, whereas the previous map ( $a p p T 1 t 1)$ [1..1000] only computes $\exp 1$ once.

The root of the problem is that the data type definition of $T$ forces us to pick one type for the constructor $M k T$. Because $\mathcal{I} \mathcal{L}$ statically links this type to an arity, we are thus forced to pick one arity for $M k T$ closures that is used throughout the entire program. $M k T$ may often be used to hold essentially binary function closures, but the computational effect of unary application of $M k T$ closures can still be crucial in certain corners of a program-either for asymptotic complexity in Haskell or for side effects in OCaml.

Rather than enforcing statically that every call is exactly saturated, perhaps we could perform a dynamic check. Given a binary application, we could interrogate the function value to check its arity, and behave differently depending on whether that arity is 1 (an over-saturated call), 2 (exactly saturated, the fast case), or greater than 2 (unsaturated). This is, in fact, what GHC does today, and corresponds to the "unknown" function calls of Marlow and Peyton Jones [2004] which inspect the arities of closure values at runtime to choose the best calling convention. But how can $\mathcal{I L}$-with its statically-tracked notion of arity-accommodate dynamic arity checking?

The key is to allow for the primitive functions contained in closures to have a different arity than their call site, thus requiring a dynamic check on all Applications of closures. Applying too few arguments creates a partial application, and applying too many is broken down into several steps. More formally, the syntax of $\mathcal{M L}$ can be extended with partial applications $\operatorname{Clos}^{n} f(\bar{a})$, where $f$ has been applied to arguments $\bar{a}$ so far and $n$ is the number of remaining arguments expected before $f$ can be called. Now, consider these extra rules for dynamic handling a runtime arity mismatch:

\begin{tabular}{|c|c|c|}
\hline (Apply) & $\left\langle\operatorname{Clos}^{n} W(\bar{a})\left|\operatorname{App}\left(\bar{a}^{\prime}\right) ; K\right| H\right\rangle \mapsto\left\langle W\left(\bar{a}, \bar{a}^{\prime}\right)|K| H\right\rangle$ & (if $\left.\left|\bar{a}^{\prime}\right|=n\right)$ \\
\hline$(P A p p)$ & $\left\langle\operatorname{Clos}^{n} W(\bar{a})\left|\operatorname{App}\left(\bar{a}^{\prime}\right) ; K\right| H\right\rangle \mapsto\left\langle\operatorname{Clos}^{n-\left|\bar{a}^{\prime}\right|} W\left(\bar{a}, \bar{a}^{\prime}\right)|K| H\right\rangle$ & (if $\left.\left|\bar{a}^{\prime}\right|<n\right)$ \\
\hline$(O A p p)$ & $\left\langle\operatorname{Clos}^{n} W(\bar{a})\left|\operatorname{App}\left(\bar{a}^{\prime}, \bar{a}^{\prime \prime}\right) ; K\right| H\right\rangle \mapsto\left\langle W\left(\bar{a}, \bar{a}^{\prime}\right)\left|\operatorname{App}\left(\bar{a}^{\prime \prime}\right) ; K\right| H\right\rangle$ & (if $\left.\left|\bar{a}^{\prime}\right|=n,\left|\bar{a}^{\prime \prime}\right|>0\right)$ \\
\hline
\end{tabular}

In practice, these extra rules for partial- and over-application lets us treat closure types like ${ }^{\mathrm{L}}\left\{\right.$ Int $\leadsto \mathrm{L}^{\mathrm{L}}$ Int $\leadsto$ Int $\left.\}\right\}$ and ${ }^{\mathrm{L}}\{$ Int $\leadsto$ Int $\leadsto$ Int $\}$ as the same, without endangering type safety due to arity mismatch. For example, this would eliminate the difference between the $T$ and $T^{\prime}$ data types from before. With these dynamic arity checks, it is safe to call appT1 $t 2^{\prime}$ : this results in a partial application because the caller (appT1) only provides one argument to the binary closure $\left(t 2^{\prime}\right)$ Likewise, it is safe to call $a p p T 2^{\prime} t 1$ : this results in an over application where the caller $\left(a p p T 2^{\prime}\right)$ wants to pass two arguments to a unary closure $(t 1)$. Yet, in the cases where the optimal arities do match (like appT1 $t 1$ and $a p p T 2^{\prime} t 2^{\prime}$ ), the fastest calling convention is used at runtime. Therefore, the types $T$ and $T^{\prime}$ can be used interchangeably, in some sense, as long as arities are checked at runtime. More generally, dynamic arity checks lets us safely equate these two closure types:

$$
\left.\left.\gamma_{\{\bar{\tau}} \leadsto \gamma_{\{\sigma\}}\right\} \gamma_{\{\bar{\tau}} \leadsto \sigma\right\}
$$

This could be formalized in $\mathcal{I} \mathcal{L}$ as a type equality or a type-safe coercion [Breitner et al. 2016]: both types are represented identically at runtime as closure objects with some runtime arity count.

In the end, both known and unknown calls of Marlow and Peyton Jones [2004] can be captured in the intermediate language. The arity of a type $\tau \leadsto \sigma$ is known statically by its kind, and the program must provide the right number of arguments and binders. However, types like $\gamma\{\tau \leadsto \sigma\}$ and $\gamma\{\tau \leadsto \gamma\{\sigma\}\}$, which both store additional arity information at runtime, can be freely interchanged at compile time, as long as the arities are checked at runtime. To be clear, this extension has a trade-off: the closures described here are subject to extra dynamic checks. It is possible that an implementation would want to have both statically checked closures and dynamically checked ones. We can accommodate both by simply having two different closure types (with their own 
Clos and App). Then, an optimizing compiler, or an expert user, can select the one with the best performance for a particular part of a program.

\section{RELATED WORK}

The system presented in this paper is the culmination of several independent lines of work on expressing performance issues directly in an intermediate language. The underlying theme is to capture the low-level details of calling conventions as features of a higher-level functional language.

\subsection{Representation and Levity in the Kinds}

The idea of distinguishing (un)boxed and (un)lifted types goes back to Peyton Jones and Launchbury [1991]. That distinction has been static until recent work added levity polymorphism to the mix [Eisenberg and Peyton Jones 2017], and shown that its utility is greater than expected (see Section 7 of that work). However, Eisenberg and Peyton Jones [2017] conflates levity polymorphism and representation polymorphism. Our contribution separates the two completely, with applications that are polymorphic in one but not the other. One our main requirements is to generate only one piece of code for every polymorphic definition. Certain definitions that must be rejected, because compilation would depend on a choice made at runtime. An alternative approach by Dunfield [2015] accepts more uses of levity polymorphism at the cost of generating different code for each choice-an exponential blowup of code size in practice-which we avoid.

\subsection{Optimizing Curried Functions}

Previous work established methods for optimizing curried function calls dynamically at runtime, avoiding the overhead of naively calling $\left(\left(\begin{array}{ll}f & 1\end{array}\right) 3\right.$ by passing one argument at a time. In practice, $f$ will often expect all three arguments before doing any interesting work, so those calls should be fused when possible. Fusing can be done by pushing many arguments on the stack at once (the push/enter model) [Krivine 2007; Leroy 1990] or by evaluating the arity of closures (the eval/apply model) [Marlow and Peyton Jones 2004]. In this work, we capture this dynamic type of optimization within the syntax and types of programs, as described in Section 7.

\subsection{Function Arity in Types}

While there is performance to be gained by dynamically optimizing curried function calls at runtime, it is even better to optimize statically at compile time. Of course, this is easy to do when the compiler can find the definition of the called function [Marlow and Peyton Jones 2004]. This scheme is easily thwarted by higher-order functions, so a less syntactic approach-like one based on types-can be beneficial. Uncurrying-representing a function $a \rightarrow b \rightarrow c \rightarrow d$ as $(a, b, c) \rightarrow d-$ is an obvious place to start, and has been investigated before [Bolingbroke and Peyton Jones 2009; Dargaye and Leroy 2009; Hannan and Hicks 1998]. However, when polymorphism is brought into the picture, type quantification is irreparably fused with multi-arity functions; see [Downen et al. 2019, Section 8.1].

Following Downen et al. [2019], $\mathcal{I} \mathcal{L}$ instead retains the curried form of function types. However, $I \mathcal{L}$ goes significantly beyond that work by supporting type polymorphism over arrow types (Section 4.3), and polymorphism over levities (Section 4.4) and conventions (Section 4.5). Another difference is that Downen et al. [2019] had two function arrows, $(\tau \stackrel{\gamma}{\rightarrow} \sigma)$ and $(\tau \leadsto \sigma)$, whereas $\mathcal{I} \mathcal{L}$ has just one arrow $(\tau \leadsto \sigma)$, plus the closure type ${ }^{\gamma}\{\tau\}$. The two are inter-convertible: we showed how to translate $\tau \stackrel{\gamma}{\rightarrow} \sigma$ to $\mathcal{I} \mathcal{L}$ for either $\gamma$ in Fig. 5, and in the other direction we have ${ }^{\gamma}\{\tau\}=() \stackrel{\gamma}{\rightarrow} \tau$ with $\left(\operatorname{Clos}^{\gamma} e\right)=\lambda^{\gamma}() . e$ and $(\operatorname{App} e)=e()$. Note that, to make the analogy operationally exact, the unit type () should be an unboxed, empty tuple (i.e., represented as 0 arguments at runtime). The approach here has a greater economy of concepts, and a nice correspondence with Int\# and Int ${ }^{\gamma}$. However, two function arrows might be better for a practical compiler. 


\subsection{The Glasgow Haskell Compiler}

GHC already implements a rich kind system, including polymorphism over types, kinds, and representations. Indeed GHC goes further: instead of a stratified zoo of different things (types, kinds, representations, etc.) as in Fig. 1, they are all types [Weirich et al. 2013] kept separate by their kinds. This is a fantastic simplification, immediately allowing polymorphism over all these conceptually-different things. This does, however, make it hard not to have polymorphism! Returning to Section 4.3, it would be hard to prevent instantiation of a forall-quantified type variable with an arrow type, requiring a restriction like "this quantified variable can have any kind other than Call." So GHC's infrastructure strongly encourages fully-fledged polymorphism.

\subsection{Logical Foundations}

The $\mathcal{I} \mathcal{L}$ language is not an ad-hoc collection of design compromises driven by only performance considerations. Rather, it grows directly from principled foundations in logic.

Previous work on unboxed types and extensional functions shares the observation that lifting-in the sense of denotational semantics-corresponds to a mismatch between machine primitives and the semantics of a programming language. Unlifted types can be implemented more directly-and therefore more efficiently-in a machine. But the cause of lifting depends on the type: unlifted integers need to be call-by-value whereas unlifted curried functions need to be call-by-name. The first reconciliation was achieved in call-by-push-value [Levy 2001], which avoids all lifting unless explicitly requested. As such, this paper can be seen as a practical extension of this foundation.

The same connection between types and evaluation is also tied to focusing and polarity [Andreoli 1992; Laurent 2002] in proof search, which corresponds to pattern-matching in functional programming [Zeilberger 2008, 2009] and semantics and computation [Munch-Maccagnoni 2009, 2013]. Recently, these mixed evaluation strategy languages have been extended with practical features like call-by-need evaluation [Downen and Ariola 2018; McDermott and Mycroft 2019] to model shared computation. Of note, the types in $\mathcal{I} \mathcal{L}$ used for boxing and indirection correspond exactly to the "polarity shifts" of Downen and Ariola [2018] to and from call-by-need. In particular, the boxed integer type corresponds to an "up shift" (Int = 介Int\#) and the function closures to a "down shift" $(\{\tau \leadsto \sigma\}=\Downarrow(\tau \leadsto \sigma))$. For the sake of usability, $\mathcal{I} \mathcal{L}$ performs other implicit polarity conversions of types based on their context. For example, closing over a non-function type like Int\# implicitly shifts it to a "nullary function" (there written $\uparrow$ Int), expressed by the encoding $\{$ Int\# $\}=\Downarrow \uparrow I n t \#$.

\section{CONCLUSION}

This paper illustrates a cohesive system for including low-level details-specifically representation, levity, and arity-inside a higher-level intermediate language. Not only does this let the language express intensional properties of programs, it also lets programs abstract over these details when they do not impact compilation. Parts of this work have been implemented already in the Glasgow Haskell Compiler, and we intend to further implement the entirety of kinds as calling conventions. The story presented here takes an explicitly typed intermediate language and-through type-driven elaboration-compiles it to an untyped target language. As future work, it could be enlightening to consider how types might be preserved by compilation by giving a sufficiently expressive type system for the lower-level language. Since the main objective is to capture performance in the intermediate language, we would also like to characterize the cost of computation in its semantics.

\section{ACKNOWLEDGMENTS}

The authors would like to thank Norman Ramsey and the anonymous reviewers, whose feedback was invaluable in improving the presentation of this paper. The material is based upon work supported by the National Science Foundation under Grant No. 1719158. 


\section{REFERENCES}

Jean-Marc Andreoli. 1992. Logic Programming with Focusing Proofs in Linear Logic. fournal of Logic and Computation 2, 3 (1992), 297-347. https://doi.org/10.1093/logcom/2.3.297

Zena M. Ariola and Matthias Felleisen. 1997. The Call-By-Need Lambda Calculus. Journal of Functional Programming 7, 3 (May 1997), 265-301. https://doi.org/10.1017/S0956796897002724

Maximilian C. Bolingbroke and Simon L. Peyton Jones. 2009. Types Are Calling Conventions. In Proceedings of the 2nd ACM SIGPLAN Symposium on Haskell (Edinburgh, Scotland) (Haskell '09). ACM, 1-12.

Joachim Breitner. 2014. Call Arity. In Trends in Functional Programming - 15th International Symposium, TFP 2014, Soesterberg, The Netherlands, May 26-28, 2014. Revised Selected Papers. 34-50.

Joachim Breitner, Richard A. Eisenberg, Simon Peyton Jones, and Stephanie Weirich. 2016. Safe zero-cost coercions for Haskell. Fournal of Functional Programming 26 (2016), e15. https://doi.org/10.1017/S0956796816000150

Zaynah Dargaye and Xavier Leroy. 2009. A verified framework for higher-order uncurrying optimizations. Higher-Order and Symbolic Computation 22, 3 (2009), 199-231.

Paul Downen and Zena M. Ariola. 2018. Beyond Polarity: Towards a Multi-Discipline Intermediate Language with Sharing. In 27th EACSL Annual Conference on Computer Science Logic, CSL 2018, September 4-7, 2018, Birmingham, UK. 21:1-21:23.

Paul Downen, Zachary Sullivan, Zena M. Ariola, and Simon Peyton Jones. 2019. Making a Faster Curry with Extensional Types. In Proceedings of the 12th ACM SIGPLAN International Symposium on Haskell (Berlin, Germany) (Haskell 2019). Association for Computing Machinery, New York, NY, USA, 58-70. https://doi.org/10.1145/3331545.3342594

Joshua Dunfield. 2015. Elaborating evaluation-order polymorphism. In Proceedings of the 20th ACM SIGPLAN International Conference on Functional Programming, ICFP 2015, Vancouver, BC, Canada, September 1-3, 2015. 256-268.

Richard Eisenberg. 2019. GHC Proposal 29: revised levity polymorphism. https://github.com/ghc-proposals/ghc-proposals/ blob/master/proposals/0029-levity-polymorphism.rst

Richard A. Eisenberg and Simon Peyton Jones. 2017. Levity polymorphism. In Proceedings of the 38th ACM SIGPLAN Conference on Programming Language Design and Implementation, PLDI 2017, Barcelona, Spain, June 18-23, 2017. 525-539.

Sebastian Graf. 2020. GHC Proposal 265: unlifted data types. https://github.com/ghc-proposals/ghc-proposals/blob/master/ proposals/0265-unlifted-datatypes.rst

John Hannan and Patrick Hicks. 1998. Higher-Order unCurrying. In POPL '98, Proceedings of the 25th ACM SIGPLAN-SIGACT Symposium on Principles of Programming Languages, San Diego, CA, USA, fanuary 19-21, 1998. 1-11.

Jean-Louis Krivine. 2007. A Call-By-Name Lambda-Calculus Machine. Higher-Order and Symbolic Computation 20, 3 (2007), 199-207.

Olivier Laurent. 2002. Étude de la polarisation en logique. Ph.D. Dissertation. Université de la Méditerranée - Aix-Marseille II.

Xavier Leroy. 1990. The ZINC experiment: an economical implementation of the ML language. Technical report 117. INRIA. Paul Blain Levy. 2001. Call-By-Push-Value. Ph.D. Dissertation. Queen Mary and Westfield College, University of London.

Simon Marlow and Simon L. Peyton Jones. 2004. Making a fast curry: push/enter vs. eval/apply for higher-order languages. In Proceedings of the Ninth ACM SIGPLAN International Conference on Functional Programming, ICFP 2004, Snow Bird, UT, USA, September 19-21, 2004. ACM, 4-15.

Andrew Martin. 2019a. GHC Proposal 112: unlifted arrays. https://github.com/ghc-proposals/ghc-proposals/blob/master/ proposals/0112-unlifted-array.rst

Andrew Martin. 2019b. GHC Proposal 203: pointer rep. https://github.com/ghc-proposals/ghc-proposals/blob/master/ proposals/0203-pointer-rep.rst

Andrew Martin. 2019c. GHC Proposal 98: unlifted newtypes. https://github.com/ghc-proposals/ghc-proposals/blob/master/ proposals/0098-unlifted-newtypes.rst

Dylan McDermott and Alan Mycroft. 2019. Extended Call-by-Push-Value: Reasoning About Effectful Programs and Evaluation Order. In Programming Languages and Systems, Luís Caires (Ed.). Springer International Publishing, Cham, 235-262.

Guillaume Munch-Maccagnoni. 2009. Focalisation and Classical Realisability. In Computer Science Logic: 23rd international Workshop, CSL 2009, 18th Annual Conference of the EACSL (Coimbra, Portugal) (CSL 2009), Erich Grädel and Reinhard Kahle (Eds.). Springer Berlin Heidelberg, Berlin, Heidelberg, 409-423.

Guillaume Munch-Maccagnoni. 2013. Syntax and Models of a non-Associative Composition of Programs and Proofs. Ph.D. Dissertation. Université Paris Diderot.

Simon L. Peyton Jones. 1992. Implementing Lazy Functional Languages on Stock Hardware: The Spineless Tagless G-machine. Journal of Functional Programming 2, 2 (1992), 127-202.

Simon L. Peyton Jones and John Launchbury. 1991. Unboxed Values As First Class Citizens in a Non-Strict Functional Language. In Proceedings of the 5th ACM Conference on Functional Programming Languages and Computer Architecture. Springer-Verlag, London, UK, UK, 636-666. 
Amr Sabry and Matthias Felleisen. 1993. Reasoning About Programs in Continuation-Passing Style. Lisp and Symbolic Computation 6, 3-4 (Nov. 1993), 289-360.

Amr Sabry and Philip Wadler. 1997. A Reflection on Call-by-Value. ACM Transactions on Programming Languages and Systems 19, 6 (1997), 916-941.

Alex Theriault. 2019. GHC Proposal 209: levity-polymorphic Lift. https://github.com/ghc-proposals/ghc-proposals/blob/ master/proposals/0209-levity-polymorphic-lift.rst

Philip Wadler, Walid Taha, and David Macqueen. 1998. How to add laziness to a strict language without even being odd. In Proceedings of the Standard ML Workshop.

Stephanie Weirich, Justin Hsu, and Richard A. Eisenberg. 2013. System FC with Explicit Kind Equality. In International Conference on Functional Programming (Boston, Massachusetts, USA) (ICFP '13). ACM.

Noam Zeilberger. 2008. On the Unity of Duality. Annals of Pure and Applied Logic 153, 1 (2008), 660-96.

Noam Zeilberger. 2009. The Logical Basis of Evaluation Order and Pattern-Matching. Ph.D. Dissertation. Carnegie Mellon University. 